\title{
Semantic Foundations for Deterministic Dataflow and Stream Processing
}

\author{
Konstantinos Mamouras \\ Rice University, Houston TX 77005, USA \\ mamouras@rice.edu
}

\begin{abstract}
We propose a denotational semantic framework for deterministic dataflow and stream processing that encompasses a variety of existing streaming models. Our proposal is based on the idea that data streams, stream transformations, and stream-processing programs should be classified using types. The type of a data stream is captured formally by a monoid, an algebraic structure with a distinguished binary operation and a unit. The elements of a monoid model the finite fragments of a stream, the binary operation represents the concatenation of stream fragments, and the unit is the empty fragment. Stream transformations are modeled using monotone functions on streams, which we call stream transductions. These functions can be implemented using abstract machines with a potentially infinite state space, which we call stream transducers. This abstract typed framework of stream transductions and transducers can be used to (1) verify the correctness of streaming computations, that is, that an implementation adheres to the desired behavior, (2) prove the soundness of optimizing transformations, e.g. for parallelization and distribution, and (3) inform the design of programming models and query languages for stream processing. In particular, we show that several useful combinators can be supported by the full class of stream transductions and transducers: serial composition, parallel composition, and feedback composition.
\end{abstract}

Keywords: Data streams · Denotational semantics · Type system

\section{Introduction}

Stream processing is the computational paradigm where the input is not presented in its entirety at the beginning of the computation, but instead it is given in an incremental fashion as a potentially unbounded sequence of elements or data items. This paradigm is appropriate in settings where data is created continually in real-time and has to be processed immediately in order to extract actionable insights and enable timely decision-making. Examples of such datasets are streams of business events in an enterprise setting [26], streams of packets that flow through computer networks [37, time-series data that is captured by sensors in healthcare applications [33], etc.

Due to the great variety of streaming applications, there are various proposals for specialized languages, compilers, and runtime systems that deal with the 
processing of streaming data. Relational database systems and SQL-based lan-

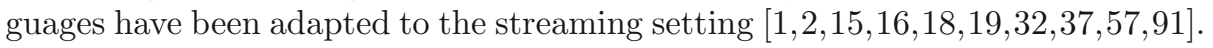
Recently, several systems have been developed for the distributed processing of data streams that are based on the distributed dataflow model of computation 6, 7, 70, 86, 92, 94, 108, 112, 113. Languages for detecting complex events in distributed systems, which draw on the theory of regular expressions and

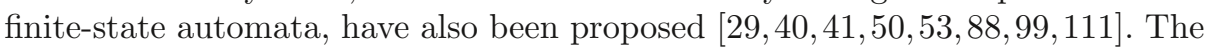
synchronous dataflow formalisms $20,24,28,51,73,107$, are based on Kahn's seminal work [59, and they have been used for exposing and exploiting tasklevel and pipeline parallelism within streaming computations in the context of embedded systems. Several formalisms for the runtime verification of reactive systems have been proposed, many of which are based on variants of Temporal Logic and its timed/quantitative extensions $39,43,52,74,105$. Finally, there is a large collection of languages and systems for reactive program-

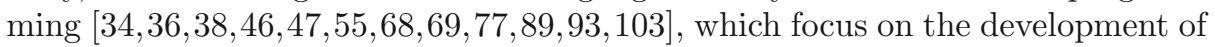
event-driven and interactive applications such as GUIs and web programming.

The aforementioned languages and systems have been successfully used in the application domains for which they were developed. However, each one of them typically introduces a unique variant of the streaming model in terms of: (1) the form of the input and output data, (2) the class of expressible stream-processing computations, and (3) the syntax employed to describe these computations. This has resulted in an enormous proliferation of semantic models for stream processing that are difficult to compare. For this reason, we are interested in identifying a semantic unification of several existing streaming models.

This paper introduces a typed semantic framework for reasoning about languages and systems for stream processing. Three key questions are tackled:

1. How do we model streams and what is the form of the data that they carry?

2. How do we capture mathematically the notion of a stream transformation?

3. What is a general programming model for specifying streaming computations?

The first two questions concern the discovery of an appropriate denotational model for streaming computation. The third question concerns the design of programming and query languages, where a key requirement is that the behavior of a streaming program/query admits a precise mathematical description. Existing works have addressed these questions in the context of specific classes of applications. Here are examples of various perspectives:

- Transductions of strings 8, 100, 104, 110]: A stream is viewed as an unbounded sequence of letters, and a stream transformation is a translation from input sequences to output sequences, which is typically called string/word transduction. These translations are commonly described using finite-state transducers, a class of automata that extend acceptors with output.

- The streaming dataflow model of Gilles Kahn 59,60]: The input and output consist of multiple independent channels that carry unbounded sequences of elements. A transformation is a function from a tuple of input sequences to a tuple of output sequences. Such transformations are specified with dataflow graphs whose nodes describe single-process computations. 
- Relational transformations 71]: A stream is an unbounded multiset (bag) of tuples, and a stream transformation is a monotone operator (w.r.t. multiset containment) on multisets. This can be generalized to consider more than one input stream. An interesting subclass of these operators can be described syntactically using monotone relational algebra.

- Processing of time-varying relations 16, 17, A stream is a timevarying finite multiset of tuples, i.e. an unbounded sequence of finite multisets of tuples. In this setting, a stream transformation processes the input in a way that preserves the notion of time: after processing $t$ input multisets (i.e., $t$ time units) the output consists of $t$ output multisets. The query language CQL [16] defines a class of such computations that involve relational and windowing operators.

- Transformations of continuous-time signals [27]: An input stream is a continuous-time signal, that is, a function from the real numbers $\mathbb{R}$ to an $n$ dimensional space $\mathbb{R}^{n}$. A stream transformation is a mapping from input signals to output signals that is causal, which means that the value of the output at time $t$ depends on the values of input signal up to (and including) time $t$. Systems of differential equations can be used to describe classes of such transformations.

We are interested here in a unifying framework that encompasses all the aforementioned concrete instances of streaming models and enables formal reasoning about the composition of streaming computations from different models. In order to achieve this we take an abstract algebraic approach that retains only the essential aspects of stream processing without any unnecessary specialization. The rest of the section outlines our proposal.

At the most fundamental level, stream processing is computation over input that is not given at the beginning in full, but rather is presented incrementally as the computation evolves. Since the input is presented piece by piece, the basic concepts that need to be captured mathematically are: (1) what is a piece or fragment of the input, and (2) how do we extend the input. The most general class of algebraic structures that model these notions is the class of monoids, the collection of algebras that have a distinguished binary associative multiplication operation · and an identity element 1 for this operation. A monoid $(A, \cdot, 1)$ then constitutes a type of data streams, where the elements of the monoid are all the possible finite stream fragments, the identity $1 \in A$ is the empty stream fragment, and the multiplication operation $\cdot: A \times A \rightarrow A$ models the concatenation of stream fragments. Using monoids, we can organize several notions of data streams using types that describe the form of the data, as well any invariants or assumptions about them. Monoids encompass the kinds of data streams that we mentioned earlier and many more: strings of letters, linear sequences of data items, tuples of sequences, multisets (bags) of data items, sets of data items, time-varying relations/multisets, (potentially disordered) timestamped sequences of data items, continuous-time signals, and so on.

Stream transformations can be classified according to the type of their input and output streams, which we call a transduction type. They are modeled using monotone functions that map an input stream history (i.e., the fragment of the input stream that has been received from the beginning of the computation 
until now) to an output stream history (i.e., the fragment of the output stream produced so far). The monotonicity requirement captures the idea that a stream transformation cannot retract the output that has already been emitted. We call such functions stream transductions, and we propose them as a denotational semantic model for stream processing. This model encompasses string transductions, non-diverging Kahn-computable [59] functions on streams, monotone relational transformations [71, the CQL-definable 16] transformations on time-varying relations, and transformations of continuous-time signals [27].

We also introduce an abstract model of computation for stream processing. The considered programs or abstract machines are called stream transducers, and they are organized using transducer types that specify the input and output stream types. A stream transducer processes the input stream in an incremental fashion, by consuming it fragment by fragment. The consumption of an input fragment results in the emission of an output fragment. Our algebraic setting brings in an unavoidable complication compared to the classical theory of word transducers: not all stream transducers describe a stream transduction. This phenomenon has to do with the generalization of the input and output data streams from sequences of atomic data items to elements of arbitrary monoids. A stream transducer has to respect its input/output type, which means that the way in which the input stream is fragmented into pieces and fed to the transducer does not affect the cumulative output. More concisely, this says that the cumulative output is independent from the fragmentation of the input. In order to formalize this notion, we say that a factorization of an input history $u$ is a sequence of stream fragments $u_{1}, u_{2}, \ldots, u_{n}$ whose concatenation is equal to the input history, i.e. $u_{1} \cdot u_{2} \cdots u_{n}=u$. Now, the desired restriction can be described as follows: for every input history $w$ and any two factorizations $u_{1}, \ldots, u_{m}$ and $v_{1}, \ldots, v_{m}$ of $w$, the cumulative output that the transducer emits when consuming the fragments $u_{1}, \ldots, u_{m}$ in sequence is equal to the cumulative output when consuming the fragments $v_{1}, \ldots, v_{n}$. Fortunately, this complex property can be distilled into an equivalent property on the structure of the stream transducer that we call coherence property. Every stream transducer that is coherent has a well-defined semantics or denotation in terms of a stream transduction.

We have already outlined the basics of our general framework for streaming computation, which includes: (1) a classification of streams using monoids as types, (2) a denotational semantic model that employs monotone functions from input histories to output histories, and (3) a programming model that generalizes transducers to compute meaninfully on elements of arbitrary monoids. This already allows us to address important questions about specific computations:

- Does a streaming program (transducer) behave as intended? This amounts to checking whether the denotation of the transducer is the desired function.

- Are two streaming programs (transducers) equivalent? This means that their denotations in terms of stream transductions are the same.

The first question is a correctness property. The second question is relevant for semantics-preserving program optimization. We will turn now to the issue of how to modularly specify complex stream transductions and transducers. 
One of the most common ways to conceptually organize complex streaming computations is to view the overall computation as the composition of several processes that run independently and are connected with directed communication channels on which streams of data flow. This way of structuring computations is called the dataflow programming model. The simple deterministic parallel model of Karp and Miller [61] is one of the first variants of dataflow, and other notable early works on dataflow models include Dennis's parallel language of actors and links [42] and Kahn's networks [59] of computing stations and communication lines. We investigate three key dataflow combinators for composing stream transductions (i.e., semantic-level) and stream transducers (i.e., program-level): serial composition, parallel composition, and feedback composition. Serial composition is useful for describing pipelines of processing stages, where the output of one stage is streamed as input into the next stage. Parallel composition describes the independent and concurrent computation of two or more components. Feedback composition supports computations whose current output depends on previously produced outputs. We show that our framework supports all these combinators, which facilitate the modular description of complex computations and expose pipeline and task-based parallelism.

Outline of paper. In Sect. 2 we introduce the idea that data streams can be classified using monoids as their types, and in Sect. 3 we propose the semantic model of stream transductions. Sect. 4 is devoted to the description of an abstract model of streaming computation, called stream transducer, and the main properties that it satisfies. In Sect. 5 we show that our abstract model is closed under a fundamental set of dataflow combinators: serial, parallel, and feedback composition. In Sect. 6 we prove the soundness of a streaming optimizing transformation using denotational arguments and algebraic rewriting. Sect. 7 contains related work, and Sect. 8 concludes with a brief summary of our proposal.

\section{Monoids as Types for Streams}

Data streams are typically viewed as unbounded linear sequences of data items, where a data item can be thought of as a small indivisible piece of data. This viewpoint is sufficient for describing many useful semantic and programming models, but it is too concrete and unnecessarily restricts the notion of a data stream. In order to see this, consider a computation where the specific order in which the data items arrive is not relevant. Counting is a trivial example of such a computation, and it can be described operationally as follows: every time a new data item arrives, the counting stream algorithm emits the total number of items that have been seen so far. This can be described mathematically by the function $\beta$, given by $\beta\left(\left\langle x_{1}, x_{2}, \ldots, x_{n}\right\rangle\right)=\langle 1,2, \ldots, n\rangle$, where $\left\langle x_{1}, x_{2}, \ldots, x_{n}\right\rangle$ is the input and $\langle 1,2, \ldots, n\rangle$ is the cumulative output of the computation. For this computation, the input can be meaningfully viewed as a multiset (or bag) instead of a sequence, since the ordering of the data items is irrelevant. This means that multisets can also be viewed as data streams, and in some cases this viewpoint is preferable to the traditional one of "streams = sequences". 
The example of the previous paragraph raises an obvious question: What class of mathematical objects can meaningfully serve as data streams? Linear sequences and multisets should certainly be included, but it would be desirable to generalize the notion of streams as much as possible. Recent works explore the idea of generalizing streams to encompass a large class of partial orders [13 85], but we will see later that this approach excludes many useful instances. Stream processing is the computational paradigm where the input is not presented in full at the beginning of the computation, but instead it is given in an incremental fashion or piece by piece. For this reason, there are just three notions that need to be modeled mathematically: (1) a fragment or piece of a data stream, (2) the extension of data with an additional fragment of data, and (3) the empty data stream, i.e. the data seen at the very beginning of the computation. This leads us to consider a kind or type of a data stream as an algebraic structure that satisfies the following: (1) its elements model data stream fragments, (2) it has a distinguished associative operation - for the concatenation of stream fragments, and (3) it has a distinguished element 1 that represents the empty fragment so that 1 is a unit for concatenation. The class of monoids is the largest class of algebraic structures that fulfill these requirements.

More formally, a monoid is an algebraic structure $(A, \cdot, 1)$, where $\cdot: A \times A \rightarrow$ $A$ is a binary operation called multiplication and $1 \in A$ is a constant called unit, that satisfies the following two axioms: (I) $(x \cdot y) \cdot z=x \cdot(y \cdot z)$ for all $x, y, z \in A$, and (II) $1 \cdot x=x \cdot 1=x$ for all $x \in A$. The first axiom says that $\cdot$ is associative, and the second axiom says that 1 is a left and right identity for the $\cdot$ operation. For brevity, we will sometimes write $x y$ to denote $x \cdot y$.

Suppose that $A$ is a monoid. We write $A^{*}$ for the set of all finite sequences of elements of $A$ and $\varepsilon$ for the empty sequence. The finite multiplication function $\pi: A^{*} \rightarrow A$ is given by $\pi(\varepsilon)=1$ and $\pi(\bar{x} \cdot\langle y\rangle)=\pi(\bar{x}) \cdot y$ for $\bar{x} \in A^{*}$ and $y \in A$. For sequences $\bar{x}, \bar{y} \in A^{*}$, it holds that $\pi(\bar{x} \cdot \bar{y})=\pi(\bar{x}) \cdot \pi(\bar{y})$. So, $\pi$ generalizes the binary multiplication to a finite but arbitrary number of arguments.

Let $\left(A, \cdot_{A}, 1_{A}\right)$ and $\left(B, \cdot_{B}, 1_{B}\right)$ be monoids. Their product is the monoid $(A \times$ $B, \cdot, 1)$, where the multiplication operation is given by $(x, y) \cdot\left(x^{\prime}, y^{\prime}\right)=(x \cdot A$ $\left.x^{\prime}, y \cdot B y^{\prime}\right)$ for $x, x^{\prime} \in A$ and $y, y^{\prime} \in B$, and the identity is $1=\left(1_{A}, 1_{B}\right)$.

A monoid homomorphism from a monoid $(A, \cdot, 1)$ to a monoid $(B, \cdot, 1)$ is a function $h: A \rightarrow B$ that commutes with the monoid operations, that is, $h(1)=1$ and $h(x \cdot y)=h(x) \cdot h(y)$ for all $x, y \in A$.

As we discussed earlier, we can think of a monoid as a type of data streams. The elements of the monoid represent finite stream fragments. The multiplication operation - models the concatenation of stream fragments, and the unit of the monoid is the empty stream fragment.

For a monoid $(A, \cdot, 1)$ we define the binary relation $\preccurlyeq$ as follows: for all $x, y \in A$, we put $x \preccurlyeq y$ if and only if $x z=y$ for some $z \in A$. Since the relation $\preccurlyeq$ is reflexive and transitive, we call it the prefix preorder for the monoid $A$. The unit 1 is a minimal element w.r.t. the $\preccurlyeq$ relation: $1 \cdot x=x$ and hence $1 \preccurlyeq x$ for every $x \in A$. Define the function prefix : $A \times A \rightarrow \mathcal{P}(A)$ as follows: $\operatorname{prefix}(x, y)=\{z \in A \mid x z=y\}$ for all $x, y \in A$. This implies that $x \preccurlyeq y$ iff 
$\operatorname{prefix}(x, y) \neq \emptyset$. In other words, $\operatorname{prefix}(x, y)$ is the set of all witnesses for $x \preccurlyeq y$. A partial function $\partial: A \times A \rightarrow A$ is said to be a prefix witness function (or simply a witness function) for the monoid $A$ if its domain is equal to $\preccurlyeq$ and it satisfies: $\partial(x, y) \in \operatorname{prefix}(x, y)$ for every $x, y \in A$ with $x \preccurlyeq y$. We can express this equivalently by requiring that the type of the function $\partial$ is $\prod_{(x, y) \in \preccurlyeq} \operatorname{prefix}(x, y)$.

We say that a monoid $A$ satisfies the left cancellation property if $x y=x z$ implies $y=z$ for all $x, y, z \in A$. In this case we say that $A$ is left-cancellative. If $A$ is left-cancellative, then it has a unique prefix witness function, because $x \preccurlyeq y$ implies that there is a unique $z$ with $x z=y$.

Example 1 (Finite Sequences). Consider the algebra $(\mathrm{FSeq}(A), \cdot, \varepsilon)$, where FSeq $(A)$ is the set $A^{*}$ of all finite words (strings) over a set $A, \cdot$ is word concatenation, and $\varepsilon$ is the empty word. This algebra is a monoid. In fact, it is the free monoid with generators $A$. For $u, v \in A^{*}, u \preccurlyeq v$ iff the word $u$ is a prefix of the word $v$. There is a unique prefix witness function, because for every $x, y \in A^{*}$ with $x \preccurlyeq y$ there is a unique $z \in A^{*}$ such that $x z=y$.

Let us consider now a variant of Example 1 in order to clear any misunderstandings regarding the $\preccurlyeq$ order. The set $A^{*}$, together with the empty sequence $\varepsilon$, and the operation $\circ$ given by $x \circ y=y x$ is a monoid. For the monoid $\left(A^{*}, \varepsilon, \circ\right)$, we have that $x \preccurlyeq y$ iff $x \circ z=z x=y$ for some $z \in A^{*}$. So, $x \preccurlyeq y$ iff the word $x$ is a suffix of the word $y$.

Example 2 (Finite Multisets). Consider the algebra $(\operatorname{FBag}(A), \cup, \emptyset)$, where $\operatorname{FBag}(A)$ is the set of all finite multisets (bags) over a set $A, \cup$ is multiset union, and $\emptyset$ is the empty multiset. This algebra is a monoid. In fact, it is the free commutative monoid with generators $A$. It is also left cancellative. For $x, y \in \mathrm{FBag}(A), x \preccurlyeq y$ iff $x$ is contained in $y$. So, we also use the notation $\subseteq$ instead of $\preccurlyeq$. There is a unique prefix witness function, because for every $x, y \in \operatorname{FBag}(A)$ with $x \subseteq y$ there is a unique $z \in \operatorname{FBag}(A)$ such that $x z=y$.

Example 3 (Finite Sets). Let $A$ be a set. Consider the algebra (FSet $(A), \cup, \emptyset)$, where $\operatorname{FSet}(A)$ is the set of all finite subsets of $A, \cup$ is set union, and $\emptyset$ is the empty set. This algebra is a monoid. In fact, it is the free commutative idempotent monoid with generators $A$. For $x, y \in \operatorname{FBag}(A), x \preccurlyeq y$ iff $x$ is contained in $y$. So, we also use the notation $\subseteq$ instead of $\preccurlyeq$.

For $x \subseteq y$, define $\partial(x, y)=y \backslash x$, where $\backslash$ is the set difference operation. Since $x \cup(y \backslash x)=y$ for $x \subseteq y, \partial$ is a prefix witness function. We also define $\tau(x, y)=y$ for $x \subseteq y$. Since $x \cup y=y$ for $x \subseteq y, \tau$ is a prefix witness function. So, $\operatorname{FSet}(A)$ has several distinct prefix witness functions.

Example 4 (Finite Maps). Let $K$ be a set of keys, and $V$ be a set of values. Consider the algebra $(\operatorname{FMap}(K, V), \cdot$,$) , where \operatorname{FMap}(K, V)$ is the set of all partial maps $K \rightarrow V$ with a finite domain, $\emptyset$ is the partial map with empty domain, and $\cdot$ is defined as follows:

$$
(f \cdot g)(k)= \begin{cases}g(k), & \text { if } g(k) \text { is defined } \\ f(k), & \text { if } g(k) \text { is undefined and } f(k) \text { is defined } \\ \text { undefined, } & \text { otherwise }\end{cases}
$$


for every $f, g \in \operatorname{FMap}(K, V)$ and $k \in K$. We leave it to the reader to check that $\emptyset \cdot f=f \cdot \emptyset=f$ and $(f \cdot g) \cdot h=f \cdot(g \cdot h)$ for all $f, g, h \in \operatorname{FMap}(K, V)$. So, the algebra $\operatorname{FMap}(K, V)$ is a monoid.

Let $f, g \in \operatorname{FMap}(A)$. We write $\operatorname{dom}(f)=\{k \in K \mid f(k)$ is defined $\}$ for the domain of $f$. It holds that $\operatorname{dom}(f \cdot g)=\operatorname{dom}(f) \cup \operatorname{dom}(g)$. Using this property, we see that $f \preccurlyeq g$ iff $\operatorname{dom}(f) \subseteq \operatorname{dom}(g)$.

Let $f, g \in \operatorname{FMap}(K, V)$ with $f \preccurlyeq g$. Define $\partial(f, g)=g$. Since $\operatorname{dom}(f) \subseteq$ $\operatorname{dom}(g)$, we have that $f \cdot \partial(f, g)=g$. It follows that $\partial$ is a prefix witness function. Define $g \backslash f \in \operatorname{FMap}(K, V)$ as follows:

$$
(g \backslash f)(k)= \begin{cases}g(k), & \text { if } g(k) \text { is defined and } f(k) \text { is undefined } \\ g(k), & \text { if } g(k), f(k) \text { are defined and } g(k) \neq f(k) \\ \text { undefined, } & \text { otherwise }\end{cases}
$$

for every $k \in K$. From $f \preccurlyeq g$ we get $f \cdot(g \backslash f)=g$. So, $\backslash$ is a prefix witness function. This means that $\operatorname{FMap}(K, V)$ has several distinct prefix witness functions.

Example 5 (Bounded-Domain Continuous-Time Signals). Let $A$ be an arbitrary set, and $\mathbb{R}$ be the set of real numbers. A bounded-domain continuoustime signal with values in $A$ is a function $f:[0, u) \rightarrow A$ where $u \geq 0$ is a real number and $[u, v)=\{t \in \mathbb{R} \mid u \leq t<v\}$. We define the concatenation operation - for such signals as follows:

$$
\frac{f:[0, u) \rightarrow A \quad g:[0, v) \rightarrow A}{f \cdot g:[0, u+v) \rightarrow A}(f \cdot g)(t)= \begin{cases}f(t), & \text { if } t \in[0, u) \\ g(t-u), & \text { if } t \in[u, u+v)\end{cases}
$$

We write $\operatorname{BSig}(A)$ for the set of all these bounded-domain continuous-time signals. The unit signal is the unique function of type $[0,0) \rightarrow A$, whose domain of definition is empty. Observe that $\operatorname{BSig}(A)$ is a monoid. For signals $f:[0, u) \rightarrow A$ and $g:[0, v) \rightarrow A$, it holds that $f \preccurlyeq g$ iff $u \leq v$ and $f(t)=g(t)$ for every $t \in[0, u)$. There is a unique prefix witness function, because for every $f, g \in \operatorname{BSig}(A)$ with $f \preccurlyeq g$ there is a unique $h \in \operatorname{BSig}(A)$ such that $f \cdot h=g$.

Example 6 (Timed Finite Sequences). We write $\mathbb{N}$ to denote the set of natural numbers (non-negative integers). A timed sequence over $A$ is an alternating sequence $s_{0} a_{1} s_{1} a_{2} \ldots a_{n} s_{n}$, where $s_{i} \in \mathbb{N}$ and $a_{i} \in A$ for every $i$. The occurrences $s_{0}, s_{1}, \ldots$ are called time punctuations and indicate the passage of time. So, the set of all timed sequences over $A$ is equal to $\operatorname{TFSeq}(A)=\mathbb{N} \cdot(A \cdot \mathbb{N})^{*}$. We define the fusion product $\diamond$ of timed sequences as follows: $s_{0} a_{1} s_{1} \ldots a_{m} s_{m} \diamond t_{0} b_{1} t_{1} \ldots b_{n} t_{n}=$ $s_{0} a_{1} s_{1} \ldots a_{m}\left(s_{m}+t_{0}\right) b_{1} t_{1} \ldots b_{n} t_{n}$. The unit timed sequence is the singleton sequence 0 . The algebra $(\operatorname{TFSeq}(A), \diamond, 0)$ is easily shown to be a monoid. There is a unique prefix witness function, because for all $x, y \in \operatorname{TFSeq}(A)$ with $x \preccurlyeq y$ there is a unique $z \in \operatorname{TFSeq}(A)$ s.t. $x \diamond z=y$.

Example 7 (Finite Time-Varying Multisets). A finite time-varying multiset over $A$ is a partial function $f: \mathbb{N} \rightarrow \operatorname{FBag}(A)$ whose domain is equal to 
$[0 . . n]=\{0, \ldots, n\}$ for some integer $n \geq 0$. We also use the notation $f:[0 . . n] \rightarrow$ $\operatorname{FBag}(A)$ to convey this information regarding the domain of $f$. We define the concatenation operation - for finite time-varying multisets as follows:

$$
\begin{array}{cl}
f:[0 . . m] \rightarrow \operatorname{FBag}(A) \\
\frac{g:[0 . . n] \rightarrow \operatorname{FBag}(A)}{f \cdot g:[0 . . m+n] \rightarrow \operatorname{FBag}(A)}
\end{array} \quad(f \cdot g)(t)= \begin{cases}f(t), & \text { if } t \in[0 . . m-1] \\
f(t) \cup g(0), & \text { if } t=m \\
g(t-m), & \text { if } t \in[m+1 . . n]\end{cases}
$$

We write $\operatorname{TFBag}(A)$ to denote the set of all finite time-varying multisets over $A$. The unit time-varying multiset Id : $[0 . .0] \rightarrow \mathrm{FBag}(A)$ is given by $\mathrm{Id}(0)=\emptyset$. It is easy to see that $f \cdot \mathbf{I d}=f$ and that $\mathbf{I d} \cdot f=f$ for every $f:[0 . . n] \rightarrow \operatorname{FBag}(A)$. We leave it to the reader to also verify that $(f \cdot g) \cdot h=f \cdot(g \cdot h)$ for finite time-varying multisets $f, g$ and $h$. So, the set $\operatorname{TFBag}(A)$ together with $\cdot$ and Id is a monoid. It is not difficult to show that it is left-cancellative.

Let us consider now the prefix preorder $\preccurlyeq$ on finite time-varying multisets. For $f:[0 . . m] \rightarrow \operatorname{FBag}(A)$ and $g:[0 . . n] \rightarrow \operatorname{FBag}(A)$, it holds that $f \preccurlyeq g$ iff $m \leq n$ and $f(t)=g(t)$ for every $t \in[0 . . m]$.

The examples above highlight the variety of mathematical objects that can be meaningfully viewed as streams. These streams can be organized elegantly using the structure of monoids. The sequences of Example 1 the multisets of Example 2 and the finite time-varying multisets of Example 7 can be described equivalently in terms of the partial orders of 13 85, which have also been suggested as an approach to unify notions of streams. Using partial orders it is also possible to model the timed finite sequences of Example 6 but only with a non-succinct encoding: every time punctuation $t \in \mathbb{N}$ is encoded with a sequence $11 \ldots 1$ of $t$ punctuations, one for each time unit. Partial orders cannot encode the sets of Example 3 the maps of Example 4 or the signals of Example 5 Informally, the reason for this is that partial orders can only encode commutation equations, which are insufficient for objects such as sets and maps.

\section{Stream Transductions}

In this section we will introduce stream transductions as semantic denotational models of stream transformations. At any given point in a streaming computation, we have seen an input history (the part of the stream from the beginning of the computation until now) and we have produced an output history (the cumulative output that has been emitted from the beginning until now). As a first approximation, a streaming computation can be described mathematically by a function $\beta: A \rightarrow B$, where $A$ and $B$ are monoids that describe the input and output type respectively, which maps an input history $x \in A$ to an output history $\beta(x) \in B$. The function $\beta$ has to be monotone because the output is cumulative, which means that it can only be extended with more output items as the computation proceeds. An equivalent way to understand the monotonicity property is that it captures the idea that any output that has already been emitted cannot be retracted. Since $\beta$ takes an entire input history as its argument, 
it can describe stateful computations, where the output that is emitted at every step potentially depends on the entire input history.

Definition 8 (Stream Transduction \& Incremental Form). Let $A$ and $B$ be monoids. A function $\beta: A \rightarrow B$ is said to be monotone (with respect to the prefix preorder) if $x \preccurlyeq y$ implies $\beta(x) \preccurlyeq \beta(y)$ for all $x, y \in A$. For a monotone $\beta: A \rightarrow B$, we say that the partial function $\mu$ is a monotonicity witness function if it maps elements $x, y \in A$ and $z \in \operatorname{prefix}(x, y)$ witnessing that $x \preccurlyeq y$ to a witness $\mu(x, y, z) \in \operatorname{prefix}(\beta(x), \beta(y))$ for $\beta(x) \preccurlyeq \beta(y)$. That is, we require that the type of $\mu$ is $\prod_{x, y \in A} \operatorname{prefix}(x, y) \rightarrow \operatorname{prefix}(\beta(x), \beta(y))$. So, the defining property of $\mu$ is that for all $x, y, z \in A$ with $x z=y$ it holds that $\beta(x) \cdot \mu(x, y, z)=\beta(y)$. For brevity, we will sometimes write $\mu(x, z)$ to denote $\mu(x, x z, z)$. The defining property of $\mu$ is then written as $\beta(x) \cdot \mu(x, z)=\beta(x z)$ for all $x, z \in A$.

A stream transduction from $A$ to $B$ is a function $\beta: A \rightarrow B$ that is monotone with respect to the prefix preorder, together with a monotonicity witness function $\mu: \prod_{x, y \in A} \operatorname{prefix}(x, y) \rightarrow \operatorname{prefix}(\beta(x), \beta(y))$. We write $\operatorname{STrans}(A, B)$ to denote the set of all stream transductions from $A$ to $B$.

The incremental form of a stream transduction $\langle\beta, \mu\rangle \in \operatorname{STrans}(A, B)$ is a function $\mathrm{F}(\beta, \mu): A^{*} \rightarrow B^{*}$, which is defined inductively by $\mathrm{F}(\beta, \mu)(\varepsilon)=\langle\beta(1)\rangle$ and $\mathrm{F}(\beta, \mu)\left(\left\langle x_{1}, \ldots, x_{n}, x_{n+1}\right\rangle\right)=\mathrm{F}(\beta, \mu)\left(\left\langle x_{1}, \ldots, x_{n}\right\rangle\right) \cdot\left\langle\mu\left(x_{1} \cdots x_{n}, x_{n+1}\right)\right\rangle$ for every sequence $\left\langle x_{1}, \ldots, x_{n+1}\right\rangle \in A^{*}$.

Consider the stream transduction $\langle\beta, \mu\rangle$ : $\operatorname{STrans}(A, B)$ and the input fragments $x, y \in A$. Notice that $\mu(x, y)$ gives the output increment that the streaming computation generates when the input history $x$ is extended into $x y$. For an arbitrary output monoid $B$, the output increment $\mu(x, y)$ is generally not uniquely determined by $\beta(x)$ and $\beta(x y)$. This means that the monotonicity witness function $\mu$ generally provides some additional information about the streaming computation that cannot be obtained purely from $\beta$. However, if the output monoid $B$ is left-cancellative then there is a unique function $\mu$ that witnesses the monotonicity of $\beta$.

Suppose that $\langle\beta, \mu\rangle: \operatorname{STrans}(A, B)$ is a stream transduction. The incremental form $\mathrm{F}(\beta, \mu)$ of the transduction $\langle\beta, \mu\rangle$ describes the stream transformation in explicit input/output increments. For example, $\mathrm{F}(\beta, \mu)\left(\left\langle x_{1}\right\rangle\right)=\left\langle\beta(1), \mu\left(1, x_{1}\right)\right\rangle$ and $\mathrm{F}(\beta, \mu)\left(\left\langle x_{1}, x_{2}\right\rangle\right)=\left\langle\beta(1), \mu\left(1, x_{1}\right), \mu\left(x_{1}, x_{2}\right)\right\rangle$. The key property of the incremental form is that $\pi(\mathrm{F}(\beta, \mu)(\bar{x}))=\beta(\pi(\bar{x}))$ for every $\bar{x} \in A^{*}$. For example, $\pi\left(\mathrm{F}(\beta, \mu)\left(\left\langle x_{1}, x_{2}, x_{3}\right\rangle\right)\right)=\beta(1) \cdot \mu\left(1, x_{1}\right) \cdot \mu\left(x_{1}, x_{2}\right) \cdot \mu\left(x_{1} x_{2}, x_{3}\right)=\beta\left(x_{1}\right) \cdot \mu\left(x_{1}, x_{2}\right)$. $\mu\left(x_{1} x_{2}, x_{3}\right)=\beta\left(x_{1} x_{2}\right) \cdot \mu\left(x_{1} x_{2}, x_{3}\right)=\beta\left(x_{1} x_{2} x_{3}\right)$.

Example 9 (Counting). Let $A$ be an arbitrary set. We will describe a streaming computation whose input type is the monoid $\operatorname{FBag}(A)$ and whose output type is the monoid FSeq $(\mathbb{N})$. The informal operational description is as follows: there is no initial output, and every time a new data item arrives the computation emits the total number of items seen so far. The formal description is given by the stream transduction $\beta: \operatorname{FBag}(A) \rightarrow \mathrm{FSeq}(\mathbb{N})$, defined by $\beta(\emptyset)=\varepsilon$ and $\beta(x)=\langle 1,2, \ldots,|x|\rangle$ for every non-empty $x \in \operatorname{FBag}(A)$, where $|x|$ denotes the size of the multiset $x$. It is easy to see that $\beta$ is monotone. Since $\operatorname{FSeq}(\mathbb{N})$ 
is left-cancellative, the monotonicity witness function is uniquely determined: $\mu(x, \emptyset)=\varepsilon$ and $\mu(x, y)=\langle|x|+1, \ldots,|x|+|y|\rangle$ when $y \neq \emptyset$.

Example 10 (Per-Key Aggregation). Let $K$ be a set of keys, and $V$ be a set of values. The elements of $K \times V$ are typically called key-value pairs. Suppose that op : $V \times V \rightarrow V$ is an associative and commutative operation. So, op can be generalized to an aggregation operation that takes non-empty finite multisets over $V$ as input. We will describe a streaming computation whose input type is the monoid $\operatorname{FBag}(K \times V)$ and whose output type is the monoid $\mathrm{FMap}(K, V)$. Informally, every time an item $(k, v)$ is processed, the output map is updated so that the $k$-indexed entry contains the aggregate (using op) of all values seen so far for the key $k$. The formal description of this computation is given by the stream transduction $\beta: \mathrm{FBag}(K \times V) \rightarrow \mathrm{FMap}(K, V)$, defined by $\beta(x)=\left\{k \mapsto \mathrm{op}\left(\left.x\right|_{k}\right) \mid k\right.$ appears in $\left.x\right\}$ for every multiset $x$, where $\left.x\right|_{k}$ denotes the multiset that results from $x$ by keeping only the pairs whose key is equal to $k$. That is, the domain of $\beta(x)$ is equal to $\operatorname{dom}(\beta(x))=\{k \in K \mid k$ appears in $x\}$ and $\beta(x)(k)=\mathrm{op}\left(\left.x\right|_{k}\right)$ for every $k$ that appears in $x$. The monotonicity witness function $\mu$ is defined as follows: $\mu(x, y)$ is equal to the restriction of the map $\beta(x \cup y)$ to the set of all keys that appear in $y$.

We saw in Sect. 2 that we can form products of monoids: if $A$ and $B$ are monoids, then so is $A \times B$. Intuitively, we can think of $A \times B$ as the data stream type that involves two parallel and independent channels: one channel for streams of type $A$ and another channel for streams of type $B$.

Example 11 (Merging of Multiple Input Channels). Given a set $A$, we want to describe a transformation with two input channels of type $\mathrm{FBag}(A)$ and one output channel of type $\operatorname{FBag}(A)$. The monotone function $\beta: \operatorname{FBag}(A) \times$ $\mathrm{FBag}(A) \rightarrow \mathrm{FBag}(A)$, given by $\beta(x, y)=x \cup y$ for multisets $x$ and $y$, describes the merging of the two input substreams. Operationally, whenever a new data item arrives (regardless of channel) it is propagated to the output channel. Since $\mathrm{FBag}(A)$ is left-cancellative, the monotonicity witness function is uniquely determined: $\mu\left(\left\langle x_{1}, y_{1}\right\rangle,\left\langle x_{2}, y_{2}\right\rangle\right)=\left(x_{2} \cup y_{2}\right) \backslash\left(x_{1} \cup y_{1}\right)$ for all $x_{1}, y_{1}, x_{2}, y_{2} \in \operatorname{FBag}(A)$.

Example 12 (Flatten). Let $A$ be a monoid. The function $\beta: \operatorname{FSeq}(A) \rightarrow A$, given by $\beta(\bar{x})=\pi(\bar{x})$ for every $\bar{x} \in \mathrm{FSeq}(A)$, describes the flattening of a sequence of monoid elements. The function $\beta$ is monotone, and its monotonicity witness function $\mu$ is given by $\mu(\bar{x}, \bar{y})=\pi(\bar{y})$ for all $\bar{x}$ and $\bar{y}$. The stream transduction flatten $(A)=\langle\beta, \mu\rangle$ has type $\operatorname{STrans}(\mathrm{FSeq}(A), A)$.

Example 13 (Split in Batches). Let $\Sigma=\{a, b\}$ be an alphabet of symbols. Suppose that we want to describe the decomposition of an element of $\Sigma^{*}$ into batches of size exactly 3 . We describe this using two functions $r_{1}$ : $\Sigma^{*} \rightarrow \mathrm{FSeq}\left(\Sigma^{*}\right)$ and $r_{2}: \Sigma^{*} \rightarrow \Sigma^{*}$. Informally, $r_{1}$ gives the sequence of full batches of size 3 , and $r_{2}$ gives the remaining incomplete batch. For example, $r_{1}(a b b a a b b a)=\langle a b b, a a b\rangle$ and $r_{2}(a b b a a b b a)=b a$.

This idea of splitting in batches can be generalized from the monoid $\Sigma^{*}$ to an arbitrary monoid $A$. We say that a splitter for $A$ is a pair $r=\left(r_{1}, r_{2}\right)$ of 
functions $r_{1}: A \rightarrow \mathrm{FSeq}(A)$ and $r_{2}: A \rightarrow A$ satisfying the following properties: (1) the equality $x=\pi\left(r_{1}(x)\right) \cdot r_{2}(x)$ says that $r_{1}$ and $r_{2}$ decompose $x \in A,(2) r_{1}\left(1_{A}\right)=\varepsilon$ says that the unit cannot be decomposed, (3) $r_{1}(x \cdot y)=$ $r_{1}(x) \cdot r_{1}\left(r_{2}(x) \cdot y\right)$ and $(4) r_{2}(x \cdot y)=r_{2}\left(r_{2}(x) \cdot y\right)$ describe how to decompose the concatenation of two monoid elements. The first two properties imply that $r_{2}\left(1_{A}\right)=1_{A}$. The third property implies that $r_{1}$ is monotone. Define $\mu(x, y)=r_{1}\left(r_{2}(x) \cdot y\right)$ for $x, y \in A$ and observe that $r_{1}(x) \cdot \mu(x, y)=r_{1}(x y)$. It follows that $\operatorname{split}(r)=\left\langle r_{1}, \mu\right\rangle$ is a stream transduction of type $\operatorname{STrans}(A, \mathrm{FSeq}(A))$.

Our denotational model of a stream transformation uses a monotone function whose domain is the monoid of (finite) input histories. We emphasize that such a denotation can also describe the transformation of an infinite stream. To illustrate this point in simple terms, consider a monotone function $\beta: A^{*} \rightarrow B^{*}$, where $A$ (resp., $B$ ) is the type of input (resp., output) items. This function extends uniquely to the $\omega$-continuous function $\beta^{\infty}: A^{\infty} \rightarrow B^{\infty}$, where $A^{\infty}=A^{*} \cup$ $A^{\omega}$ is the set of finite and infinite sequences over $A$, as follows: $\beta^{\infty}\left(a_{0} a_{1} a_{2} \ldots\right)$ is equal to the supremum of the chain $\beta(\varepsilon) \leq \beta\left(a_{0}\right) \leq \beta\left(a_{0} a_{1}\right) \leq \ldots$

\section{Model of Computation}

We will present an abstract model of computation for stream processing, where the input and output data streams are elements of monoids $A$ and $B$ respectively. A streaming algorithm is described by a transducer, a kind of automaton that produces output values. We consider transducers that can have a potentially infinite state space, which we denote by St. The computation starts at a distinguished initial state init $\in \mathrm{St}$, and the initialization triggers some initial output $\mathrm{o} \in B$. The computation then proceeds by consuming the input stream incrementally, i.e. fragment by fragment. One step of the computation from a state $s \in$ St involves consuming an input fragment $x \in A$, producing an output increment out $(s, x) \in B$ and transitioning to the next state next $(s, x) \in$ St.

Definition 14 (Stream Transducer). Let $A, B$ be monoids. A stream transducer with inputs from $A$ and outputs from $B$ is a tuple $\mathcal{G}=$ (St, init, o, next, out), where $\mathrm{St}$ is a nonempty set of states, init $\in \mathrm{St}$ is the initial state, $\mathrm{o} \in B$ is the initial output, next : $\mathrm{St} \times A \rightarrow \mathrm{St}$ is the transition function, and out: $\mathrm{St} \times A \rightarrow B$ is the output function. We write $\mathrm{G}(A, B)$ to denote the set of all stream transducers with inputs from $A$ and outputs from $B$.

We define the generalized transition function gnext : St $\times A^{*} \rightarrow$ St by induction: $\operatorname{gnext}(s, \varepsilon)=s$ and $\operatorname{gnext}(s,\langle x\rangle \cdot \bar{y})=\operatorname{gnext}(\operatorname{next}(s, x), \bar{y})$ for all $s \in \mathrm{St}$, $x \in A$ and $\bar{y} \in A^{*}$. A state $s \in \mathrm{St}$ is said to be reachable in $\mathcal{G}$ if there exists a sequence $\bar{x} \in A^{*}$ such that gnext(init, $\left.\bar{x}\right)=s$.

We define the generalized output function gout : St $\times A^{*} \rightarrow B$ by induction on the second argument: $\operatorname{gout}(s, \varepsilon)=1$ and $\operatorname{gout}(s,\langle x\rangle \cdot \bar{y})=\operatorname{out}(s, x)$. $\operatorname{gout}(\operatorname{next}(s, x), \bar{y})$ for all $s \in \mathrm{St}, x \in A$ and $\bar{y} \in A^{*}$. The extended output function eout : St $\times A^{*} \rightarrow B^{*}$ is defined similarly: eout $(s, \varepsilon)=\varepsilon$ and eout $(x,\langle x\rangle \cdot \bar{y})=$ $\langle\operatorname{out}(s, x)\rangle \cdot \operatorname{eout}(\operatorname{next}(s, x), \bar{y})$ for all $s \in \mathrm{St}, x \in A$ and $\bar{y} \in A^{*}$. 
Example 15 (Transducer for Counting). Recall the counting streaming computation that was described in Example 9. We will describe a stream transducer that implements the counting computation. The input monoid is $\operatorname{FBag}(A)$ and the output monoid is $\mathrm{FSeq}(\mathbb{N})$. The state space is $\mathrm{St}=\mathbb{N}$, because the transducer has to maintain a counter that remembers the number of data items seen so far. The initial state is init $=0$ and the initial output is $o=\varepsilon$. The transition function increments the counter, i.e. next $(s, x)=s+|x|$ for every $s \in \mathrm{St}$ and $x \in \mathrm{FBag}(A)$. The output function is defined by out $(s, \emptyset)=\varepsilon$ and out $(s, x)=\langle s+1, \ldots, s+|x|\rangle$ for a nonempty multiset $x$. The type of this transducer is $\mathrm{G}(\mathrm{FBag}(A), \mathrm{FSeq}(\mathbb{N}))$.

Example 16 (Transducer for Merging). We will implement the merging computation of Example 11, where there are two input channels of type $\operatorname{FBag}(A)$ and one output channel of type $\operatorname{FBag}(A)$. The transducer does not need memory, so St $=$ Unit, where Unit $=\{\star\}$ is a singleton set. The initial state is init $=\star$ and the initial output is $0=\emptyset$. There is only one possibility for the transition function: $\operatorname{next}(s,\langle x, y\rangle)=\star$. The output function describes the propagation of the input increments of both input channels to the output channel: out $(s,\langle x, y\rangle)=x \cup y$ for all multisets $x, y$. The type of this transducer is $\mathrm{G}(\mathrm{FBag}(A) \times \mathrm{FBag}(A), \operatorname{FBag}(A))$.

Example 17 (Flatten). For a monoid $A$, we define a transducer Flatten $(A)=$ (St, init, o, next, out) : $\mathrm{G}(\mathrm{FSeq}(A), A)$ that implements the flattening transduction of Example 12. This computation does not require memory, so we define St $=$ Unit and init $=\star$. The initial output is $0=1_{A}$, the transition function is uniquely determined by next $(s, x)=\star$, and the output function is given by $\operatorname{out}\left(s,\left\langle a_{1}, \ldots, a_{n}\right\rangle\right)=a_{1} \cdots a_{n}$.

Example 18 (Split in Batches). For a monoid $A$ and a splitter $r=\left(r_{1}, r_{2}\right)$ for $A$ (Example 13), we describe a transducer Split $(r)=(\mathrm{St}$, init, o, next, out) that implements the transduction split( $r): \operatorname{STrans}(A, \operatorname{FSeq}(A))$. We define $\mathrm{St}=A$, because the transducer needs to remember the remainder of the cumulative input that does not yet form a complete batch, and init $=1_{A}$. The initial output $\mathrm{o}=\varepsilon$ is the empty sequence. The transition and output functions are defined by $\operatorname{next}(s, x)=r_{2}(s \cdot x)$ and out $(s, x)=r_{1}(s \cdot x)$.

Definition 14 does not capture a key requirement for streaming computations over monoids, namely that the cumulative output of a transducer $\mathcal{G}$ should be independent of the particular way in which the input history is split into the fragments that are fed to it. More precisely, suppose that $w$ is an input history that can be fragmented (factorized) in two different ways: $w=u_{1} \cdot u_{2} \cdots u_{m}$ and $w=v_{1} \cdot v_{2} \cdots v_{n}$. Then, the cumulative output of the transducer $\mathcal{G}$ when consuming the sequence of fragments (factorization) $u_{1}, u_{2}, \ldots, u_{m}$ should be equal to the cumulative output when consuming $v_{1}, v_{2}, \ldots, v_{n}$. In Definition 20 below, we formulate a set of coherence conditions that a transducer must adhere to in order to satisfy this "factorization independence" requirement. 
Definition 19 (Bisimulation \& Bisimilarity). Let $\mathcal{G}=$ (St, init, o, next, out) be a transducer with inputs from $A$ and outputs from $B$. A relation $R \subseteq \mathrm{St} \times \mathrm{St}$ is a bisimulation for $\mathcal{G}$ if for every $s, t \in \mathrm{St}$ and $x \in A$ we have that $(s, t) \in R$ implies out $(s, x)=\operatorname{out}(t, x)$ and $(\operatorname{next}(s, x), \operatorname{next}(t, x)) \in R$. We will also use the notation $s R t$ to mean $(s, t) \in R$. We say that the states $s, t \in R$ are bisimilar, denoted $s \sim t$, if there exists a bisimulation $R$ for $\mathcal{G}$ such that $s R t$. The relation $\sim$ is called the bisimilarity relation for $\mathcal{G}$.

It is well-known that the bisimilarity relation for $\mathcal{G}$ is an equivalence relation (reflexive, symmetric, and transitive), and for all $s, t \in \mathrm{St}$ and $x \in A$ it satisfies the following extension property: $s \sim t$ implies that next $(s, x) \sim \operatorname{next}(t, x)$. It can then be easily seen that the bisimilarity relation is a bisimulation. In fact, it is the largest bisimulation for the transducer $\mathcal{G}$.

Definition 20 (Coherence). Suppose $\mathcal{G}=($ St, init, o, next, out) : $\mathrm{G}(A, B)$ is a stream transducer. We say that $\mathcal{G}$ is coherent if it satisfies the following:

(N1) $\operatorname{next}($ init, 1$) \sim$ init.

(N2) next(init, $x y) \sim \operatorname{next}(\operatorname{next}($ init, $x), y)$ for every $x, y \in A$.

(O1) o. out (init, 1) $=$ o.

$(\mathrm{O} 2) \mathrm{o} \cdot \operatorname{out}($ init, $x y)=\mathrm{o} \cdot \operatorname{out}($ init, $x) \cdot \operatorname{out}(\operatorname{next}($ init, $x), y)$ for every $x, y \in A$.

The coherence conditions of Definition 20 capture the idea that the transducer behaves in "essentially the same way" regardless of how the input is split into fragments. For example, the condition (N2) says that the two-step transition init $\rightarrow^{x} s_{1} \rightarrow^{y} s_{2}$ and the single-step transition init $\rightarrow^{x y} t_{1}$ end up in states $\left(s_{2}\right.$ and $\left.t_{1}\right)$ that will have exactly the same behavior in the subsequent computation. In other words, it does not matter whether the input $x y$ was fed to the transducer as a single fragment $x y$ or as a sequence of two fragments $\langle x, y\rangle$.

Let $(A, \cdot, 1)$ be a monoid. A factorization of an element $x \in A$ is a sequence $x_{1}, \ldots, x_{n}$ of elements of $A$ such that $x=x_{1} \cdots x_{n}$. In particular, the empty sequence $\varepsilon \in A^{*}$ is a factorization of 1 . In other words, $\bar{x} \in A^{*}$ is a factorization of $x \in A$ if $\pi(\bar{x})=x$.

Theorem 21 (Factorization Independence). Let $\mathcal{G}=$ (St, init, o, next, out) be a stream transducer of type $\mathrm{G}(A, B)$. If $\mathcal{G}$ is coherent, then for every $x \in A$ and every factorization $\bar{x} \in A^{*}$ of $x$ we have that $\mathrm{o} \cdot \operatorname{gout}($ init, $\bar{x}$ ) $=0 \cdot$ out(init, $x$ ).

Proof. For clarity, we write $\left\langle x_{1}, x_{2}, \ldots, x_{n}\right\rangle \in A^{*}$ to denote a finite sequence of elements of $A$. The following properties hold for all $s \in \mathrm{St}, \bar{x} \in A^{*}$ and $y \in A$ :

$$
\begin{aligned}
\operatorname{gnext}(s, \bar{x} \cdot\langle y\rangle) & =\operatorname{next}(\operatorname{gnext}(s, \bar{x}), y) \\
\operatorname{gout}(s, \bar{x} \cdot\langle y\rangle) & =\operatorname{gout}(s, \bar{x}) \cdot \operatorname{out}(\operatorname{gnext}(s, \bar{x}), y) \\
\operatorname{eout}(s, \bar{x} \cdot\langle y\rangle) & =\operatorname{eout}(s, \bar{x}) \cdot\langle\operatorname{out}(\operatorname{gnext}(s, \bar{x}), y)\rangle
\end{aligned}
$$

Each property shown above can be proved by induction on the sequence $\bar{x}$.

Consider an arbitrary coherent stream transducer $\mathcal{G}=$ (St, init, o, next, out). We claim that $\mathcal{G}$ satisfies the following coherence property:

$$
\text { gnext(init, } \left.\left\langle x_{1}, \ldots, x_{n}\right\rangle\right) \sim \operatorname{next}\left(\text { init, } x_{1} \cdots x_{n}\right) \text { for all }\left\langle x_{1}, \ldots, x_{n}\right\rangle \in A^{*} \text {. }
$$


The proof is by induction on the length of the sequence. For the base case, we have that gnext(init, $\varepsilon)=$ init and next(init, 1) are bisimilar because $\mathcal{G}$ is coherent (recall Property (N1) of Definition 20). For the induction step we have:

$$
\begin{aligned}
\operatorname{gnext}(\text { init, } \bar{x} \cdot\langle y\rangle) & =\operatorname{next}(\operatorname{gnext}(\text { init, } \bar{x}), y) \\
& \sim \operatorname{next}(\operatorname{next}(\text { init, } \pi(\bar{x})), y) \\
& \sim \operatorname{next}(\operatorname{init}, \pi(\bar{x}) \cdot y),
\end{aligned}
$$

[Equation (1)]

[I.H., extension]

[coherence (N2)]

which is equal to next(init, $\pi(\bar{x} \cdot\langle y\rangle))$. This concludes the proof of the claim $\left(\mathrm{N}^{*}\right.$.

The proof of the theorem proceeds by induction on $\bar{x} \in A^{*}$. For the base case, observe that $\mathrm{o} \cdot \operatorname{gout}($ init, $\varepsilon)=0 \cdot 1=\mathrm{o}$ is equal to $\mathrm{o} \cdot \mathrm{out}($ init, 1$)=\mathrm{o}$ (property $(\mathrm{O} 1)$ for $\mathcal{G})$. For the induction step, we have:

$$
\begin{aligned}
\mathrm{o} \cdot \operatorname{gout}(\text { init, } \bar{x} \cdot\langle y\rangle) & =0 \cdot \operatorname{gout}(\text { init, } \bar{x}) \cdot \operatorname{out}(\operatorname{gnext}(\text { init, } \bar{x}), y) \\
& =0 \cdot \operatorname{out}(\text { init, } \pi(\bar{x})) \cdot \operatorname{out}(\operatorname{gnext}(\text { init, } \bar{x}), y) \\
& =0 \cdot \operatorname{out}(\text { init, } \pi(\bar{x})) \cdot \operatorname{out}(\operatorname{next}(\text { init, } \pi(\bar{x})), y) \\
& =0 \cdot \operatorname{out}(\text { init, } \pi(\bar{x}) \cdot y)
\end{aligned}
$$

which is equal to $0 \cdot$ out(init, $\pi(\bar{x} \cdot\langle y\rangle))$.

Theorem 21 says that the condition of coherence guarantees a basic correctness property for stream transducers: the output that they produce does not depend on the specific way in which the input was partitioned into fragments.

For a transducer $\mathcal{G}=\left(\right.$ St, init, o, next, out) we define the function $\llbracket G \rrbracket: A^{*} \rightarrow$ $B^{*}$ as follows: $\llbracket G \rrbracket(\bar{x})=\langle$ o $\rangle \cdot \operatorname{eout}($ init, $\bar{x})$ for every $\bar{x} \in A^{*}$. We call $\llbracket \mathcal{G} \rrbracket$ the interpretation or denotation of $\mathcal{G}$. The definition of $\llbracket \mathcal{G} \rrbracket$ implies that $\llbracket \mathcal{G} \rrbracket(\varepsilon)=$ $\langle o\rangle$ and the following holds for every $\bar{x} \in A^{*}$ and $y \in A$ :

$$
\llbracket \mathcal{G} \rrbracket(\bar{x} \cdot\langle y\rangle)=\llbracket \mathcal{G} \rrbracket(\bar{x}) \cdot\langle\text { out }(\text { gnext }(\text { init }, \bar{x}), y)\rangle
$$

When $\mathcal{G}$ is coherent, Theorem 21 says that the denotation gives the same cumulative output for any two factorizations of the input. We say that the transducers $\mathcal{G}_{1}$ and $\mathcal{G}_{2}$ are equivalent if their denotations are equal, i.e. $\llbracket \mathcal{G}_{1} \rrbracket=\llbracket \mathcal{G}_{2} \rrbracket$.

Definition 22 (The Implementation Relation). Let $A, B$ be monoids, $\mathcal{G}$ : $\mathrm{G}(A, B)$ be a stream transducer, and $\langle\beta, \mu\rangle: \operatorname{STrans}(A, B)$ be a stream transduction. We say that $\mathcal{G}$ implements $\langle\beta, \mu\rangle$ if $\llbracket \mathcal{G} \rrbracket(\bar{x})=\mathrm{F}(\beta, \mu)(\bar{x})$ for every $\bar{x} \in A^{*}$.

Theorem 23 (Implementation \& Coherence). A stream transducer $\mathcal{G}$ : $\mathrm{G}(A, B)$ is coherent if and only if it implements some stream transduction.

Proof. Suppose that $\mathcal{G}=($ St, init, o, next, out) : $\mathrm{G}(A, B)$ is a coherent transducer. Define the function $\beta: A \rightarrow B$ by $\beta(x)=0$. out(init, $x$ ) for every $x \in A$, and the function $\mu: A \times A \rightarrow B$ by $\mu(x, y)=\operatorname{out}($ next(init, $x), y)$ for all $x, y \in$ $A$. For any $x, y \in A$, we have to establish that $\beta(x) \cdot \mu(x, y)=\beta(x y)$. This follows immediately from Part (O2) of the coherence property for $\mathcal{G}$. So, $\langle\beta, \mu\rangle$ is a stream transduction. It remains to prove that $\mathcal{G}$ implements $\langle\beta, \mu\rangle$, that is, 
$\llbracket \mathcal{G} \rrbracket(\bar{x})=\mathrm{F}(\beta, \mu)(\bar{x})$ for every $\bar{x} \in A^{*}$. For the base case, we have $\llbracket \mathcal{G} \rrbracket(\varepsilon)=\langle 0\rangle$ and $\mathrm{F}(\beta, \mu)(\varepsilon)=\langle\beta(1)\rangle$, which are equal because $\beta(1)=\mathrm{o} \cdot \mathrm{out}$ (init, 1$)=\mathrm{o}$ by (O1). For the step case, we observe that:

$$
\begin{aligned}
\llbracket \mathcal{G} \rrbracket(\bar{x} \cdot\langle y\rangle) & =\llbracket \mathcal{G} \rrbracket(\bar{x}) \cdot\langle\operatorname{out}(\operatorname{gnext}(\text { init, } \bar{x}), y)\rangle & & {[\text { Equation } 4] } \\
\mathrm{F}(\beta, \mu)(\bar{x} \cdot\langle y\rangle) & =\mathrm{F}(\beta, \mu)(\bar{x}) \cdot\langle\mu(\pi(\bar{x}), y)\rangle & & {[\text { def. of } \mathrm{F}(\beta, \mu)] }
\end{aligned}
$$

By the induction hypothesis, it suffices to show that out(gnext(init, $\bar{x}), y$ ) is equal to $\mu(\pi(\bar{x}), y)=\operatorname{out}(\operatorname{next}($ init, $\pi(\bar{x})), y)$. This follows from the fact that gnext(init, $\bar{x}$ ) and next(init, $\pi(\bar{x}))$ are bisimilar, see Property $\mathbf{N}^{*}$.

For the converse, suppose that $\mathcal{G}=($ St, init, o, next, out) : $\mathrm{G}(A, B)$ is a transducer that implements $\langle\beta, \mu\rangle: \operatorname{STrans}(A, B)$. Define the relation $R$ as:

$$
\begin{array}{r}
R=\{(s, t) \in \mathrm{St} \times \mathrm{St} \mid \\
\qquad s=\operatorname{gnext}(\text { init, } \bar{x}) \text { and } t=\operatorname{gnext}(\text { init, } \bar{y})\} .
\end{array}
$$

We claim that $R$ is a bisimulation. Consider arbitrary states $s, t \in$ St with $s R t$ and $z \in A$. It follows that there are $\bar{x}, \bar{y} \in A^{*}$ with $\pi(\bar{x})=\pi(\bar{y})$ such that $s=\operatorname{gnext}($ init, $\bar{x})$ and $t=\operatorname{gnext}($ init, $\bar{y})$. We have to show that $\operatorname{out}(s, z)=\operatorname{out}(t, z)$ and $\operatorname{next}(s, z) R \operatorname{next}(t, z)$. First, notice that:

$$
\begin{aligned}
\llbracket \mathcal{G} \rrbracket(\bar{x} \cdot\langle z\rangle) & =\llbracket \mathcal{G} \rrbracket(\bar{x}) \cdot\langle\operatorname{out}(s, z)\rangle & & \text { [Equation } 44, \text { def. of } s] \\
\mathrm{F}(\beta, \mu)(\bar{x} \cdot\langle z\rangle) & =\mathrm{F}(\beta, \mu)(\bar{x}) \cdot\langle\mu(\pi(\bar{x}), z)\rangle & & {[\text { def. of } \mathrm{F}(\beta, \mu)] }
\end{aligned}
$$

Since $\mathcal{G}$ implements $\langle\beta, \mu\rangle$, we have that $\llbracket \mathcal{G} \rrbracket(\bar{x} \cdot\langle z\rangle)=\mathrm{F}(\beta, \mu)(\bar{x} \cdot\langle z\rangle)$ and therefore out $(s, z)=\mu(\pi(\bar{x}), z)$. Similarly, we can obtain that out $(t, z)=\mu(\pi(\bar{y}), z)$. From $\pi(\bar{x})=\pi(\bar{y})$ we get that $\mu(\pi(\bar{x}), z)=\mu(\pi(\bar{y}), z)$, and therefore out $(s, z)=$ out $(t, z)$. Now, observe that $s^{\prime}=\operatorname{next}(s, z)=\operatorname{next}(\operatorname{gnext}($ init, $\bar{x}), z)=\operatorname{gnext}(\bar{x}$. $\langle z\rangle)$ using Property 1. Similarly, we have that $t^{\prime}=\operatorname{next}(t, z)=\operatorname{gnext}(\bar{y} \cdot\langle z\rangle)$. From $\pi(\bar{x} \cdot\langle z\rangle)=\pi(\bar{x}) z=\pi(\bar{y}) z=\pi(\bar{y} \cdot\langle z\rangle)$ we conclude that $s^{\prime} R t^{\prime}$. We have thus established that $R$ is a bisimulation.

Now, we are ready to prove that $\mathcal{G}$ is coherent. We will only present the cases of Part (N2) and Part (O2), since they are the most interesting ones. Let $x, y \in A$. For Part (N2), we have to show that the states $s=\operatorname{next}(\operatorname{next}($ init, $x), y$ ) and $t=\operatorname{next}($ init, $x y$ ) are bisimilar. Since $R$ (previous paragraph) is a bisimulation, it suffices to show that $(s, t) \in R$. Indeed, this is true because $s=\operatorname{gnext}($ init, $\langle x, y\rangle)$, $t=\operatorname{gnext}($ init,$\langle x y\rangle)$ and $\pi(\langle x, y\rangle)=x y=\pi(\langle x y\rangle)$. For Part (O2), we have that $\llbracket G \rrbracket(\langle x y\rangle)=\langle$ o, out (init, $x y)\rangle$ and $\mathrm{F}(\beta, \mu)(\langle x y\rangle)=\langle\beta(1), \mu(1, x y)\rangle$, as well as

$$
\begin{aligned}
\llbracket \mathcal{G} \rrbracket(\langle x, y\rangle) & =\langle\mathrm{o}, \text { out }(\text { init }, x), \text { out }(\operatorname{next}(\text { init }, x), y)\rangle \text { and } \\
\mathrm{F}(\beta, \mu)(\langle x, y\rangle) & =\langle\beta(1), \mu(1, x), \mu(x, y)\rangle,
\end{aligned}
$$

using the definitions of $\llbracket G \rrbracket$ and $\mathrm{F}$. Since $\mathcal{G}$ implements $\langle\beta, \mu\rangle$, we know that $\llbracket \mathcal{G} \rrbracket(\langle x, y\rangle)=\mathrm{F}(\beta, \mu)(\langle x, y\rangle)$ and $\llbracket G \rrbracket(\langle x y\rangle)=\mathrm{F}(\beta, \mu)(\langle x y\rangle)$. Using all the above, we get that $\mathrm{o} \cdot \operatorname{out}($ init, $x) \cdot \operatorname{out}($ next(init, $x), y)=\beta(1) \cdot \mu(1, x) \cdot \mu(x, y)=\beta(x)$. $\mu(x, y)=\beta(x y)$ and $\mathrm{o} \cdot \mathrm{out}($ init, $x y)=\beta(1) \cdot \mu(1, x y)=\beta(x y)$. So, Part (O2) of the coherence property holds. 
Theorem 23 provides justification for our definition of the coherence property for stream transducers (recall Definition 20. It says that the definition is exactly appropriate, because it is a necessary and sufficient condition for a stream transducer to have a stream transduction as its denotation. In other words, the coherence property characterizes the transducers have a well-defined denotational semantics in terms of transductions. It offers this guarantee of correctness without limiting their expressive power as implementations of transductions.

Theorem 24 (Expressive Completeness). Let $A$ and $B$ be monoids, and $\langle\beta, \mu\rangle$ be a stream transduction in $\operatorname{STrans}(A, B)$. There exists a coherent stream transducer that implements $\langle\beta, \mu\rangle$.

Proof. Recall from Definition 8 that the monotonicity witness function $\mu$ satisfies the following property: $\beta(x) \cdot \mu(x, y)=\beta(x y)$ for every $x, y \in A$. Now, we define the transducer $\mathcal{G}=($ St, init, o, next, out) as follows: St $=A$, init $=1, \mathrm{o}=\beta(1)$, $\operatorname{next}(s, x)=s \cdot x$ and out $(s, x)=\mu(s, x)$ for every state $s \in$ St and input $x \in A$. The following properties hold for every $s \in$ St and $\left\langle x_{1}, \ldots, x_{n}\right\rangle \in A^{*}$ :

$$
\begin{aligned}
\operatorname{gnext}\left(s,\left\langle x_{1}, \ldots, x_{n}\right\rangle\right) & =s \cdot x_{1} \cdots x_{n} \quad \text { and } \\
\langle\mathrm{o}\rangle \cdot \operatorname{eout}\left(\text { init },\left\langle x_{1}, \ldots, x_{n}\right\rangle\right) & =\mathrm{F}(\beta, \mu)\left(\left\langle x_{1}, \ldots, x_{n}\right\rangle\right)
\end{aligned}
$$

Both these properties are shown by induction on the sequence $\left\langle x_{1}, \ldots, x_{n}\right\rangle$. It follows that $\llbracket G \rrbracket(\bar{x})=\langle$ o $\rangle \cdot$ eout $($ init, $\bar{x})=\mathrm{F}(\beta, \mu)(\bar{x})$ for every $\bar{x} \in A^{*}$. So, $\mathcal{G}$ implements the transduction $\langle\beta, \mu\rangle$. Finally, $\mathcal{G}$ is coherent by Theorem 23

Theorem 24 assures us that the abstract computational model of coherent stream transducers is expressive enough to implement any stream transduction. For this reason, we will be using stream transducers as the basic programming model for describing streaming computations.

Example 25 (Correctness of Flatten). Using induction, we will show that the transducer $\mathcal{G}=$ Flatten $(A)=$ (Unit, $\star, 1_{A}$, next, out) implements the transduction $\langle\pi, \mu\rangle=\operatorname{flatten}(A)$ for a monoid $A$ (recall Examples 12 and 17 ). We show by induction that $\llbracket \mathcal{G} \rrbracket(\bar{x})=\mathrm{F}(\pi, \mu)(\bar{x})$ for every $\bar{x} \in \mathrm{FSeq}(A)^{*}$. For the base case, we have that $\llbracket \mathcal{G} \rrbracket(\varepsilon)=\left\langle 1_{A}\right\rangle$ and $\mathrm{F}(\pi, \mu)(\varepsilon)=\langle\pi(\varepsilon)\rangle=\left\langle 1_{A}\right\rangle$. Now,

$$
\begin{aligned}
\llbracket \mathcal{G} \rrbracket(\bar{x} \cdot\langle y\rangle) & =\llbracket \mathcal{G} \rrbracket(\bar{x}) \cdot\langle\operatorname{out}(\operatorname{gnext}(\text { init, } \bar{x}), y)\rangle & & \text { [def. of } \llbracket \mathcal{G} \rrbracket] \\
& =\mathrm{F}(\pi, \mu)(\bar{x}) \cdot\langle\pi(y)\rangle & & {[\text { I.H. and def. }} \\
& =\mathrm{F}(\pi, \mu)(\bar{x}) \cdot\langle\mu(\pi(\bar{x}), y)\rangle & & \text { [def. of } \mu] \\
& =\mathrm{F}(\pi, \mu)(\bar{x} \cdot\langle y\rangle) & & \text { [def. of } \mathrm{F}]
\end{aligned}
$$

for all $\bar{x} \in \mathrm{FSeq}(A)^{*}$ and $y \in \mathrm{FSeq}(A)$. We have thus proved that $\mathrm{Flatten}(A)$ is correct: its denotation is equal to the intended semantics.

Example 26 (Correctness of Split). We will establish that the transducer for splitting in batches is correct, namely that $\mathcal{G}=\operatorname{Split}(r)=\left(A, 1_{A}, \varepsilon\right.$, next, out $)$ implements $\left\langle r_{1}, \mu\right\rangle=\operatorname{split}(r)$ for a splitter $r=\left(r_{1}, r_{2}\right)$ for the monoid $A$ (recall 
Examples 13 and 18. Using the properties of splitters and an argument by induction, we obtain that gnext(init, $\bar{x})=r_{2}(\pi(\bar{x}))$ for every $\bar{x} \in A^{*}$. We show by induction that $\llbracket \mathcal{G} \rrbracket(\bar{x})=\mathrm{F}\left(r_{1}, \mu\right)(\bar{x})$ for every $\bar{x} \in A^{*}$. For the base case, we have that $\llbracket \mathcal{G} \rrbracket(\varepsilon)=\langle\varepsilon\rangle$ and $\mathrm{F}\left(r_{1}, \mu\right)(\varepsilon)=\left\langle r_{1}\left(1_{A}\right)\right\rangle=\langle\varepsilon\rangle$. Now,

$$
\begin{aligned}
\llbracket \mathcal{G} \rrbracket(\bar{x} \cdot\langle y\rangle) & =\llbracket \mathcal{G} \rrbracket(\bar{x}) \cdot\langle\operatorname{out}(\operatorname{gnext}(\text { init, } \bar{x}), y)\rangle & & {[\text { Equation } 4] } \\
& =\mathrm{F}\left(r_{1}, \mu\right)(\bar{x}) \cdot\left\langle\operatorname{out}\left(r_{2}(\pi(\bar{x})), y\right)\right\rangle & & {[\text { I.H. and previous claim] }} \\
& =\mathrm{F}\left(r_{1}, \mu\right)(\bar{x}) \cdot\left\langle r_{1}\left(r_{2}(\pi(\bar{x})) \cdot y\right)\right\rangle & & {[\text { def. of out] }} \\
& =\mathrm{F}\left(r_{1}, \mu\right)(\bar{x}) \cdot\langle\mu(\pi(\bar{x}), y)\rangle & & {[\text { def. of } \mu] } \\
& =\mathrm{F}\left(r_{1}, \mu\right)(\bar{x} \cdot\langle y\rangle) & & {[\text { def. of } \mathrm{F}] }
\end{aligned}
$$

for all $\bar{x} \in A^{*}$ and $y \in A$. We have thus established that $\operatorname{Split}(r)$ is correct: its denotation is equal to the intended semantics.

\section{Combinators for Deterministic Dataflow}

We consider four dataflow combinators: (1) the lifting of pure morphisms to streaming computations, (2) serial composition for exposing pipeline parallelism, (3) parallel composition for exposing task-based parallelism, and (4) feedback composition for describing computations whose current output depends on previously produced output. The combinators are defined both for stream transductions (semantic objects) and for stream transducers (programs). Table 1 shows the definitions. The lifting of pure morphisms is implemented with a stateless transducer (i.e., the state space is a singleton set). Both parallel and serial composition are implemented using a product construction on transducers. In the case of parallel composition, each component computes independently. In the case of serial composition, the output of the first component is passed as input to the second component. In the case of feedback composition, the computation proceeds in well-defined rounds in order to prevent divergence.

We prove a precise correspondence between the semantics-level and programlevel combinators for all cases: lifting (Proposition 27), parallel composition (Propsition 28), serial composition (Proposition 29), and feedback composition (Proposition 30 . These are essentially correctness properties for the implementations of the combinators Lift, Par, Serial, Loop. They establish that our typed framework is appropriate for the modular specification of complex streaming computations, as it can support composition constructs that are essential for parallelization and distribution.

Proposition 27 (Lifting). Let $h: A \rightarrow B$ be a monoid homomorphism. Then, $\operatorname{Lift}(h)$ is a coherent transducer and it implements the transduction $\operatorname{lift}(h)$.

Proposition 28 (Parallel Composition). Let $A_{1}, A_{2}, B_{1}, B_{2}$ be monoids, $\left\langle\beta_{1}, \mu_{1}\right\rangle$ : $\operatorname{STrans}\left(A_{1}, B_{1}\right)$ and $\left\langle\beta_{2}, \mu_{2}\right\rangle: \operatorname{STrans}\left(A_{2}, B_{2}\right)$ be transductions, and $\mathcal{G}_{1}: \mathrm{G}\left(A_{1}, B_{1}\right)$ and $\mathcal{G}_{2}: \mathrm{G}\left(A_{2}, B_{2}\right)$ be transducers.

(1) Implementation: If $\mathcal{G}_{1}$ implements $\left\langle\beta_{1}, \mu_{1}\right\rangle$ and $\mathcal{G}_{2}$ implements $\left\langle\beta_{2}, \mu_{2}\right\rangle$, then $\operatorname{Par}\left(\mathcal{G}_{1}, \mathcal{G}_{2}\right)$ implements $\left\langle\beta_{1}, \mu_{1}\right\rangle \|\left\langle\beta_{2}, \mu_{2}\right\rangle$. 
Table 1. Combinators for deterministic dataflow.

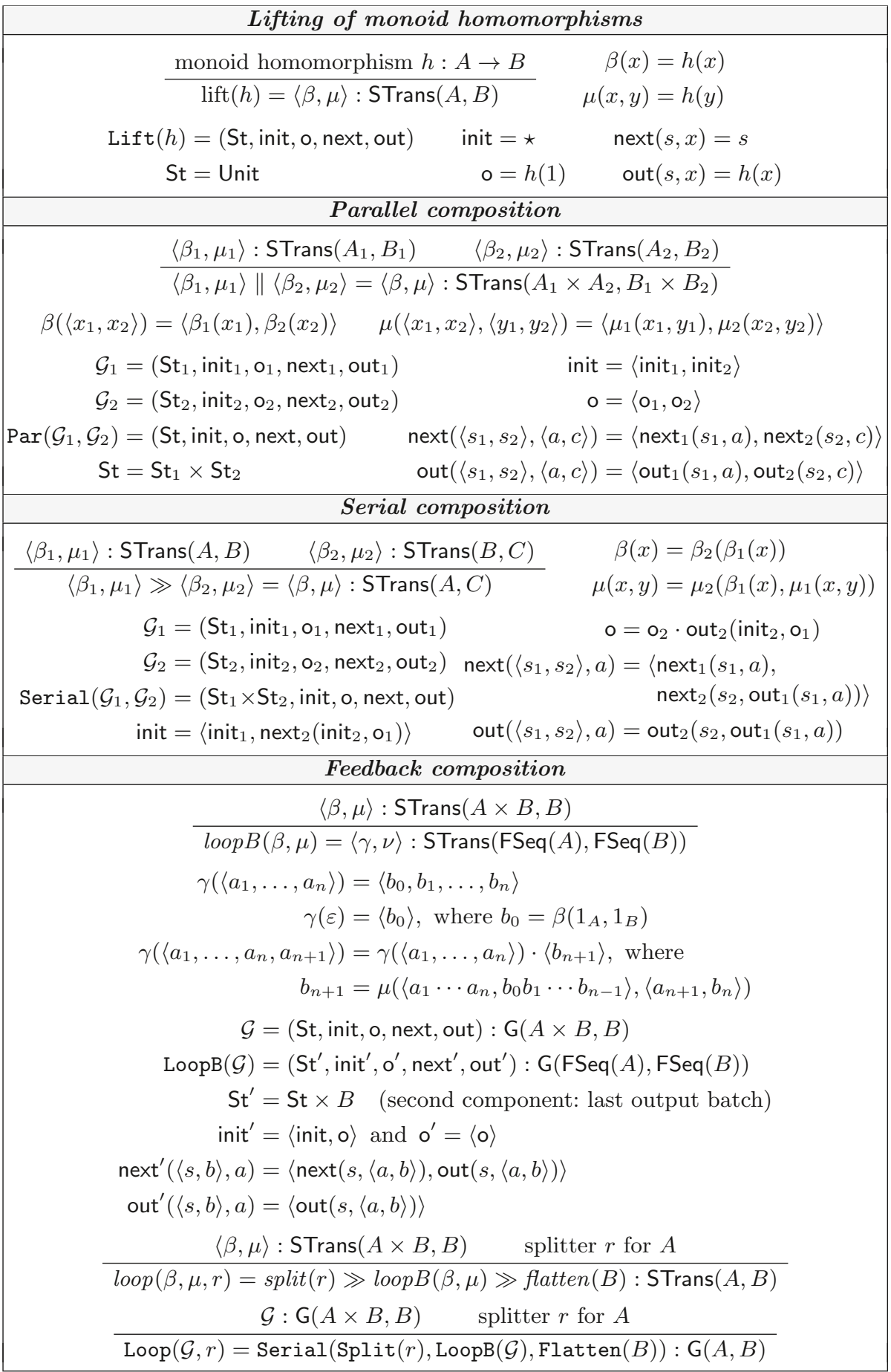


(2) Coherence: If $\mathcal{G}_{1}$ and $\mathcal{G}_{2}$ are coherent, then so is $\operatorname{Par}\left(\mathcal{G}_{1}, \mathcal{G}_{2}\right)$.

Proof. Notice that Part (2) follows immediately from Part (1) and Theorem 23 Define $f=\llbracket \operatorname{Par}\left(\mathcal{G}_{1}, \mathcal{G}_{2}\right) \rrbracket$ and $\langle\beta, \mu\rangle=\left\langle\beta_{1}, \mu_{1}\right\rangle \|\left\langle\beta_{2}, \mu_{2}\right\rangle$. We will show that $f(\bar{w})=\mathrm{F}(\beta, \mu)(\bar{w})$ for every $\bar{w} \in\left(A_{1} \times A_{2}\right)^{*}$. Suppose that fst is the (elementwise) left projection function. We claim that $\operatorname{fst}(\operatorname{gnext}(s, \bar{w}))=\operatorname{gnext}_{1}(\operatorname{fst}(s), \operatorname{fst}(\bar{w}))$ and $\operatorname{fst}(\operatorname{eout}(s, \bar{w}))=\operatorname{eout}_{1}(\mathrm{fst}(s)$, fst $(\bar{w}))$ for all $s \in$ St and $\bar{w} \in\left(A_{1} \times A_{2}\right)^{*}$. Both claims are shown by induction on the length of $\bar{w}$. With similar arguments we can obtain that $\operatorname{snd}(f(\bar{w}))=\llbracket \mathcal{G}_{2} \rrbracket(\operatorname{snd}(\bar{w}))$ for every $\bar{w} \in\left(A_{1} \times A_{2}\right)^{*}$. It can be shown by induction that $\mathrm{fst}(\mathrm{F}(\beta, \mu)(\bar{w}))=\mathrm{F}\left(\beta_{1}, \mu_{1}\right)(\mathrm{fst}(\bar{w}))$ and $\operatorname{snd}(\mathrm{F}(\beta, \mu)(\bar{w}))=$ $\mathrm{F}\left(\beta_{1}, \mu_{1}\right)(\operatorname{snd}(\bar{w}))$ for all $\bar{w} \in\left(A_{1} \times A_{2}\right)^{*}$. In order to establish that $f(\bar{w})=$ $\mathrm{F}(\beta, \mu)(\bar{w})$, it suffices to show that $\mathrm{fst}(f(\bar{w}))=\mathrm{fst}(\mathrm{F}(\beta, \mu)(\bar{w}))$ and $\operatorname{snd}(f(\bar{w}))=$ $\operatorname{snd}(\mathrm{F}(\beta, \mu)(\bar{w}))$. Given the claims shown previously, these equalities are equivalent to $\llbracket \mathcal{G}_{1} \rrbracket(\operatorname{fst}(\bar{w}))=\mathrm{F}\left(\beta_{1}, \mu_{1}\right)($ fst $(\bar{w}))$ and $\llbracket \mathcal{G}_{2} \rrbracket(\operatorname{snd}(\bar{w}))=\mathrm{F}\left(\beta_{2}, \mu_{2}\right)(\operatorname{snd}(\bar{w}))$ respectively. These equalities follow from the assumptions that $\mathcal{G}_{1}$ implements $\left\langle\beta_{1}, \mu_{1}\right\rangle$ and $\mathcal{G}_{2}$ implements $\left\langle\beta_{2}, \mu_{2}\right\rangle$.

Proposition 29 (Serial Composition). Let $A, B, C$ be monoids, $\left\langle\beta_{1}, \mu_{1}\right\rangle$ : $\operatorname{STrans}(A, B)$ and $\left\langle\beta_{2}, \mu_{2}\right\rangle$ : $\operatorname{STrans}(B, C)$ be transductions, and $\mathcal{G}_{1}: \mathrm{G}(A, B)$ and $\mathcal{G}_{2}: \mathrm{G}(B, C)$ be transducers.

(1) Implementation: If $\mathcal{G}_{1}$ implements $\left\langle\beta_{1}, \mu_{1}\right\rangle$ and $\mathcal{G}_{2}$ implements $\left\langle\beta_{2}, \mu_{2}\right\rangle$, then $\operatorname{Serial}\left(\mathcal{G}_{1}, \mathcal{G}_{2}\right)$ implements $\left\langle\beta_{1}, \mu_{1}\right\rangle \gg\left\langle\beta_{2}, \mu_{2}\right\rangle$.

(2) Coherence: If $\mathcal{G}_{1}$ and $\mathcal{G}_{2}$ are coherent, then so is $\operatorname{Serial}\left(\mathcal{G}_{1}, \mathcal{G}_{2}\right)$.

Proof. Part (2) follows easily from Part (1) and Theorem 23. In order to prove Part (1) we have to first establish a number of preliminary facts. We define the function $\mathrm{M}_{2}: A^{*} \rightarrow A$ as follows: $\mathrm{M}_{2}(\varepsilon)=1, \mathrm{M}_{2}(\langle x\rangle)=x$ for $x \in A$, and $\mathrm{M}_{2}(\langle x, y\rangle \cdot \bar{z})=\langle x y\rangle \cdot \bar{z}$ for $x, y \in A$ and $\bar{z} \in A^{*}$. We write $\mathcal{G}$ to denote $\mathcal{G}_{1} \gg \mathcal{G}_{2}$.

$$
\begin{aligned}
\operatorname{fst}(\operatorname{gnext}(s, \bar{x})) & =\operatorname{gnext}_{1}(\mathrm{fst}(s), \bar{x}) \text { for all } s \in \mathrm{St} \text { and } \bar{x} \in A^{*} \\
\operatorname{snd}(\operatorname{gnext}(s, \bar{x})) & =\operatorname{gnext}_{2}\left(\operatorname{snd}(s), \text { eout }_{1}(\mathrm{fst}(s), \bar{x})\right) \text { for all } s \in \text { St and } \bar{x} \in A^{*} \\
\llbracket \mathcal{G} \rrbracket(\bar{x}) & =\mathrm{M}_{2}\left(\llbracket \mathcal{G}_{2} \rrbracket\left(\llbracket \mathcal{G}_{1} \rrbracket(\bar{x})\right)\right) \text { for all } \bar{x} \in A^{*} \\
\mathrm{~F}(\beta, \mu)(\bar{x}) & =\mathrm{M}_{2}\left(\mathrm{~F}\left(\beta_{2}, \mu_{2}\right)\left(\mathrm{F}\left(\beta_{1}, \mu_{1}\right)(\bar{x})\right)\right) \text { for all } \bar{x} \in A^{*}
\end{aligned}
$$

where $\langle\beta, \mu\rangle=\left\langle\beta_{1}, \mu_{1}\right\rangle \gg\left\langle\beta_{2}, \mu_{2}\right\rangle$. All four claims above are proved by induction on the sequence $\bar{x}$. Equations (7) and (8) are needed to prove Equation (9). Now, we will establish that $\mathcal{G}$ implements $\langle\beta, \mu\rangle$. Indeed, we have that

$$
\begin{aligned}
\llbracket \mathcal{G} \rrbracket(\bar{x}) & =\mathrm{M}_{2}\left(\llbracket \mathcal{G}_{2} \rrbracket\left(\llbracket \mathcal{G}_{1} \rrbracket(\bar{x})\right)\right) & & {[\text { Equation }[9]] } \\
& =\mathrm{M}_{2}\left(\llbracket \mathcal{G}_{2} \rrbracket\left(\mathrm{F}\left(\beta_{1}, \mu_{1}\right)(\bar{x})\right)\right) & & {\left[\mathcal{G}_{1} \text { implements }\left\langle\beta_{1}, \mu_{1}\right\rangle\right] } \\
& =\mathrm{M}_{2}\left(\mathrm{~F}\left(\beta_{2}, \mu_{2}\right)\left(\mathrm{F}\left(\beta_{1}, \mu_{1}\right)(\bar{x})\right)\right) & & {\left[\mathcal{G}_{2} \text { implements }\left\langle\beta_{2}, \mu_{2}\right\rangle\right] } \\
& =\mathrm{F}(\beta, \mu)(\bar{x}) & & {[\text { Equation }[10]}
\end{aligned}
$$

for every $\bar{x} \in A^{*}$. So, we conclude that $\mathcal{G}$ implements $\langle\beta, \mu\rangle$.

Let us give an example of how to construct complex computations from simpler ones using the dataflow combinators. Let $A, B$ be sets and op : $A \rightarrow B$ 
be a function. We want to describe a streaming computation with two input channels, both of type $\mathrm{FBag}(A)$, and one output channel of type $\operatorname{FBag}(B)$. The computation transforms both input channels in the same way, namely by applying the function op to each element. This gives two output substreams, both of type $\operatorname{FBag}(B)$, that are merged into the output stream. The function op : $A \rightarrow B$ lifts to a monoid homomorphism op : $\mathrm{FBag}(A) \rightarrow \mathrm{FBag}(B)$, given by $\mathrm{op}(x)=\{\mathrm{op}(a) \mid a \in x\}$ for every multiset $x$. The streaming computation described previously can be visualized using the dataflow graph shown below.

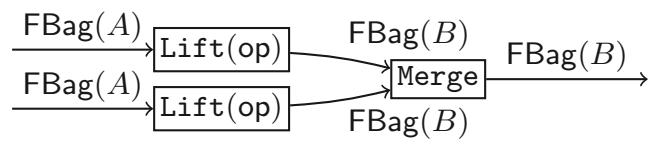

Each edge of the graph represents a communication channel along which a stream flows, and it is annotated with the type of the stream. The dataflow graph above represents the transducer $\mathcal{G}=\operatorname{Serial}(\operatorname{Par}(\operatorname{Lift}(\mathrm{op})$, Lift(op)), Merge), where Merge : $\mathrm{G}(\mathrm{FBag}(A) \times \mathrm{FBag}(A), \mathrm{FBag}(A))$ is the transducer of Example 16 From Propositions 27, 29 and 28 we obtain that $\mathcal{G}$ implements the transduction (lift(op) \| lift(op)) $\gg$ merge, where merge is described in Example 11

We will now consider the feedback combinator, which introduces cycles in the dataflow graph. One consequence of cyclic graphs in the style of KahnMacQueen 60 is that divergence can be introduced, that is, a finite amount of input can cause an operator to enter an infinite loop. For example, consider the transducer Merge : $\mathrm{G}(\mathrm{FBag}(A) \times \operatorname{FBag}(A), \operatorname{FBag}(A))$ of Example 16 . The figure below visualizes the dataflow graph, where the output channel of Merge is connected to one of its input channels, thus forming a feedback loop.

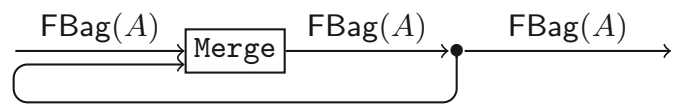

Suppose that the singleton input $\{a\}$ is fed to the input of the dataflow graph above, which corresponds to the first input channel of Merge. This will cause Merge to emit $\{a\}$, which will be sent again to the second input channel of Merge. Intuitively, this will cause the computation to enter an infinite loop (divergence) of consuming and emitting $\{a\}$. This behavior is undesirable in systems that process data streams, because divergence can make the system unresponsive. For this reason, we will consider here a form of feedback that eliminates this problem by ensuring that the computation of a feedback loop proceeds in a sequence of rounds. This avoid divergence, because the computation always makes progress by moving from one round to the next, as dictated by the input data. We describe this organization in rounds by requiring that the programmer specifies a splitter (recall Example 18). The splitter decomposes the input stream into batches, and one round of computation for the feedback loop corresponds to consuming one batch of data, generating the corresponding output batch, and sending the output batch along the feedback loop to be available for the next round of processing. This form of feedback allows flexibility in specifying what constitutes 
a single batch (and thus a single round), and therefore generalizes the feedback combinator of Synchronous Languages such as Lustre 31.

Proposition 30 (Feedback Composition). Let $A$ and $B$ be monoids, $\langle\beta, \mu\rangle$ : $\operatorname{STrans}(A, B)$ be a transduction, $\mathcal{G}: \mathrm{G}(A, B)$ be a transducer, and $r=\left(r_{1}, r_{2}\right)$ be a splitter for $A$ (see Example 13 .

(1) Implem.: If $\mathcal{G}$ implements $\langle\beta, \mu\rangle$, then $\operatorname{Loop}(\mathcal{G}, r)$ implements $\operatorname{loop}(\beta, \mu, r)$.

(2) Coherence: If $\mathcal{G}$ is coherent, then so is $\operatorname{Loop}(\mathcal{G}, r)$.

Proof. We leave to the reader the proofs that Split (Example 18) implements split and that Flatten (Example 17) implements flatten. Given Proposition 29. it suffices to show that $\mathcal{G}^{\prime}=\operatorname{LoopB}(\mathcal{G})$ implements $\langle\gamma, \nu\rangle=\operatorname{loop} B(\beta, \mu)$. Since $\mathcal{G}^{\prime}$ is of type $\mathrm{G}(\mathrm{FSeq}(A), \mathrm{FSeq}(B))$ it suffices to define the transition and output functions on singleton sequences (as done in Table 1), because there is a unique way to extend them so that $\mathcal{G}^{\prime}$ is coherent. It remains to show that $\llbracket \mathcal{G}^{\prime} \rrbracket(\bar{x})=$ $\mathrm{F}(\gamma, \nu)(\bar{x})$ for every $\bar{x} \in \mathrm{FSeq}(A)^{*}$. The base case is easy, and for the step case it suffices to show that out' $\left(\right.$ gnext $^{\prime}\left(\right.$ init $\left.\left.^{\prime}, \bar{x}\right), y\right)=\nu(\pi(\bar{x}), y)$ for every $\bar{x} \in \mathrm{FSeq}(A)^{*}$ and $y \in \operatorname{FSeq}(A)$. As we discussed before, gnext ${ }^{\prime}$ and out ${ }^{\prime}$ can be viewed as being defined on elements of $A$ rather than sequences of FSeq $(A)$, so we can equivalently prove that out' gnext' $^{\prime}$ init $\left.\left.^{\prime},\left\langle a_{1}, \ldots, a_{n}\right\rangle\right), a_{n+1}\right)=\nu\left(\left\langle a_{1}, \ldots, a_{n}\right\rangle, a_{n+1}\right)$ with each $a_{i}$ an element of $A$. Given that $\mathcal{G}$ implements $\langle\beta, \mu\rangle$, the key observation to finish the proof is $\operatorname{gnext}^{\prime}\left(\right.$ init $\left.^{\prime},\left\langle a_{1}, \ldots, a_{n}\right\rangle\right)=\left\langle\operatorname{gnext}\left(\right.\right.$ init,$\left.\left.\left\langle\left\langle a_{1}, b_{0}\right\rangle, \ldots,\left\langle a_{n}, b_{n-1}\right\rangle\right\rangle\right), b_{n}\right\rangle$, where $\gamma\left(\left\langle a_{1}, \ldots, a_{n}\right\rangle\right)=\left\langle b_{0}, b_{1}, \ldots, b_{n}\right\rangle$.

Example 31. For an example of using the feedback combinator, consider the transduction $\langle\beta, \mu\rangle$ which adds two input streams of numbers pointwise. That is, $\beta: \mathrm{FSeq}(\mathbb{N}) \times \mathrm{FSeq}(\mathbb{N}) \rightarrow \mathrm{FSeq}(\mathbb{N})$ is defined by $\beta\left(x_{1} x_{2} \ldots x_{m}, y_{1} y_{2} \ldots y_{n}\right)=$ $0\left(x_{1}+y_{1}\right)\left(x_{2}+y_{2}\right) \ldots\left(x_{k}+y_{k}\right)$ where $k=\min (m, n)$. Additionally, consider the trivial splitter $r=\left(r_{1}, r_{2}\right)$ for sequences where each batch is a singleton: $r_{1}\left(x_{1} \ldots x_{n}\right)=\left\langle x_{1}, \ldots, x_{n}\right\rangle$ and $r_{2}\left(x_{1} \ldots x_{n}\right)=\varepsilon$. We use this splitter to enforce that each batch is a single element and that each round of the computation involves consuming one element. Finally, the transduction $\operatorname{loo} p(\beta, \mu, r)=\langle\gamma, \nu\rangle$ describes the running sum, that is, $\gamma\left(x_{1} \ldots x_{n}\right)=0 x_{1}\left(x_{1}+x_{2}\right) \ldots\left(x_{1}+\cdots+x_{n}\right)$.

The dataflow combinators of this section could form the basis of query lan-

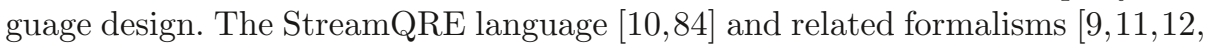
14 are based on a set of combinators for efficiently processing linearly-ordered streams (e.g., time series 3, 4]). Extending a language like StreamQRE to the typed setting of stream transductions is an interesting research direction.

\section{Algebraic Reasoning for Optimizing Transformations}

Our typed denotational framework can be used to validate optimizing transformations using algebraic reasoning. This amounts to establishing that the original transducer is equivalent to the optimized one. A fundamental approach for showing equivalence of composite transducers is to establish algebraic laws between basic building blocks, and then use algebraic rewriting. 
As a concrete example, consider the per-key streaming aggregation of Example 10, which is described by the transduction $\operatorname{reduce}(K$, op $): \operatorname{STrans}(\operatorname{FBag}(K \times$ $V), \operatorname{FMap}(K, V))$, where $K$ is the set of keys, $V$ is the set of values, and op : $V \times V \rightarrow V$ is an associative and commutative aggregation operation. Let $h: K \rightarrow\{1, \ldots, n\}$ be a hash function for the keys, and define $K_{i}^{h}=h^{-1}(i)=$ $\{k \in K \mid h(k)=i\}$ for every $i$. Consider two variants of the merging operation of Example 11: (1) kmerge $(h)$ merges $n$ input streams of types $\operatorname{FBag}\left(K_{1}^{h} \times V\right), \ldots$, $\operatorname{FBag}\left(K_{n}^{h} \times V\right)$ respectively into an output stream of type $\operatorname{FBag}(K \times V)$, and (2) mmerge $(h)$ merges $n$ input streams of types $\operatorname{FMap}\left(K_{1}^{h}, V\right), \ldots, \operatorname{FMap}\left(K_{n}^{h}, V\right)$ respectively into an output stream of type $\operatorname{FMap}(K, V)$. We also consider the transduction $\operatorname{ksplit}(h)$ that partitions an input stream of type $\operatorname{FBag}(K \times V)$ into $n$ output substreams of types $\operatorname{FBag}\left(K_{1}^{h} \times V\right), \ldots, F \operatorname{Fag}\left(K_{n}^{h} \times V\right)$ respectively. Using elementary set-theoretic arguments, the following equalities can be established: $\operatorname{ksplit}(h) \gg \operatorname{kmerge}(h)=i d$ and

$$
\operatorname{kmerge}(h) \gg \operatorname{rd}(K, \text { op })=\left(\operatorname{rd}\left(K_{1}^{h}, \text { op }\right)\|\cdots\| \operatorname{rd}\left(K_{n}^{h}, \text { op }\right)\right) \gg \operatorname{mmerge}(h),
$$

where rd abbreviates reduce. Next, we consider the corresponding transducers KSplit $(h)$, KMerge $(h)$, Id, Reduce (K, op) (abbreviation Rd) and MMerge $(h)$ and establish that they implement the respective transductions. This can be shown with induction proofs as shown earlier in Example 25 and Example 26. Using these facts and the propositions of Sect. 5 the equalities between transductions shown earlier give the following equations (equivalences) between transducers: $\operatorname{KSplit}(h) \gg \operatorname{KMerge}(h) \equiv \mathrm{Id}$ and

$$
\operatorname{KMerge}(h) \gg \operatorname{Rd}(K, \text { op }) \equiv\left(\operatorname{Rd}\left(K_{1}^{h}, \text { op }\right)\|\cdots\| \operatorname{Rd}\left(K_{n}^{h}, \text { op }\right)\right) \gg \operatorname{MMerge}(h) .
$$

Using these equations, we can establish the following optimizing transformation for data parallelization, which is useful when processing high-rate data streams.

$$
\begin{aligned}
\operatorname{Reduce}(K, \text { op }) & \equiv \mathrm{Id} \gg \operatorname{Reduce}(K, \text { op }) \\
& \equiv \operatorname{KSplit}(h) \gg \operatorname{KMerge}(h) \gg \operatorname{Reduce}(K, \text { op }) \\
& \equiv \operatorname{KSplit}(h) \gg\left(\operatorname{Rd}\left(K_{1}^{h}, \text { op }\right)\|\cdots\| \operatorname{Rd}\left(K_{n}^{h}, \text { op }\right)\right) \gg \operatorname{MMerge}(h) .
\end{aligned}
$$

The above equation illustrates our proposed style of reasoning for establishing the soundness of optimizing streaming transformations: (1) prove equalities between transductions using elementary set-theoretic arguments, (2) prove that the transducers (programs) implement the transductions (denotations) using induction, (3) translate the equalities between transductions into equivalences between transducers using the results of Sect. 5, and finally (4) use algebraic reasoning to establish more complex equivalences.

The example of this section is simple but illustrates two key points: (1) our data types for streams (monoids) capture important invariants about the streams that enable transformations, and (2) useful program transformations can be established with denotational arguments that require an appropriate notion of transduction. This approach opens up the possibility of formally verifying the wealth of optimizing transformations that are used in stream processing systems. 
The papers 54,101] describe several of them, but use informal arguments that rely on the operational intuition about streaming computations. Our approach here, on the other hand, relies on rigorous denotational arguments.

The equational axiomatizations of arrows $[56$ and traced monoidal categories [58] are relevant to our setting, but would require adaptation. An interesting question is whether a complete axiomatization can be provided for the basic dataflow combinators of Sect. 5, similarly to how Kleene Algebra (KA) 62, 63 and its extensions $\left.\begin{array}{|l|l|l|l|l|l|l|l|l|l|}\hline 49 & 64 & 79 & 83 & \text { (as well as other program logics } & 65 & 66 & 78 & 80 & 82\end{array}\right]$ capture properties of imperative programs at the propositional level. We also leave for future work the development of the coalgebraic approach 96 98. for reasoning about the equivalence of stream transducers. We have already defined a notion of bisimulation in Sect. 4, which could give an alternative approach for proving equivalence using coinduction on the transducers.

\section{Related Work}

Sect. 1 contains several pointers to related literature for stream processing. In this section, we will focus on prior work that specifically addresses aspects of formal semantics for streaming computation.

The seminal work of Gilles Kahn [59] is exemplary in its rigorous treatment of denotational semantics for a language of deterministic dataflow graphs of independent processes, which access their input channels using blocking read statements and the output channels using nonblocking write statements. The language Lustre 31] is a synchronous restriction of Kahn's model, which introduces the semantic idea of a clock for specifying the rate of a stream. Other notable synchronous formalisms are the language Signal [21 72 ] and Esterel [22 28], and the synchronous dataflow graphs of 73 and 24 . These formalisms are all deterministic, in the sense the the output is determined purely by the input data. Nondeterminism creates unavoidable semantic complications [30].

The CQL language 16 is a streaming extension of a relational database language with additional constructs for time-based windowing. The denotational semantics of CQL [17] can be reconstructed and greatly simplified within our framework using the notion of stream described in Example 7 (finite time-varying multisets). There are several works that deal with the semantics of specific language constructs (e.g., windows), notions of time, punctuations and disordered streams, but do not give a mathematical description of the overall streaming computation $5,7,25,44,67,75,76,109$.

The literature on Functional Reactive Programming ( FRP $34,46,47,55$, $68,69,93,103$ is closely related to the deterministic dataflow formalisms mentioned earlier. The main abstractions in FRP are signals and event sequences, which are linearly ordered data. Processing unordered data (e.g., multisets and maps) and extracting data parallelism (e.g., the per-key aggregation of Sect. 6) require a data model that goes beyond linear orders. In particular, the axioms of arrows 56. (often used in FRP) cannot prove the soundness of the optimizing transformation of Sect. 6] which requires reasoning about multisets. 
The idea of using types to classify streams has been recently explored in 85 (see also 13 ), but only for a restricted class of types that correspond to partial orders. No general abstract model of computation is presented in [85], and many of the examples in this paper cannot be adequately accomodated.

The mathematical framework of coalgebras 97] has been used to describe streams 98 . One advantage of this approach is that proofs of equivalence can be given using the proof principle of coinduction [96], which in many cases offers a useful alternative to proofs by induction. This line of work mostly focuses on infinite sequences of elements, whereas here we focus on the transformation of streams of data that can be of various different forms (not just sequences).

The idea to model the input/output of automata using monoids has appeared in the algebraic theory of automata and transducers. Monoids (non-free, e.g. $A^{*} \times B^{*}$ ) have been used to generalize automata from recognizers of languages to recognizers of relations 45, which are sometimes called rational transducers 100]. Our focus here is on (deterministic) functions, as models that recognize relations can give rise to the Brock-Ackerman anomaly [30]. The automata models (with inputs from a free monoid $A^{*}$ ) most closely related to our stream transducers are deterministic: Mealy machines [87, Moore machines [90], sequential transducers [4, 95], and sub-sequential transducers 102. The concept of coherence that we introduce here (Definition 20 does not arise in these models, because they do not operate on input batches. An algebraic generalization of a deterministic acceptor is provided by a right monoid action $\delta: \mathrm{St} \times A \rightarrow \mathrm{St}$ (see page 231 of 100 ), which satisfies the following properties for all $s \in$ St and $x, y \in A:(1) \delta(s, 1)=s$, and $(2) \delta(\delta(s, x), y)=\delta(s, x y)$. These properties look similar to (N1) and (N2) of Definition 20 They are, however, too restrictive for our stream transducers, as they would falsify Theorem 23

\section{Conclusion}

We have presented a typed semantic framework for stream processing, based on the idea of abstracting data streams as elements of algebraic structures called monoids. Data streams are thus classified using monoids as types. Stream transformations are modeled as monotone functions, which are organized by input/output type. We have adapted the classical model of string transducers to our setting, and we have developed a general theory of streaming computation with a formal denotational semantics. The entire technical development in this paper is constructive, and therefore lends itself well to formalization in a proof assistant such as Coq $23,35,106$. Our framework can be used for the formalization of streaming models, and the validation of subtle optimizations of streaming programs (e.g., Sect. 6), such as the ones described in 54,101]. We have restricted our attention in this paper to deterministic streaming computation, in the sense that the behaviors that we model have predictable and reproducible results. Nondeterminism causes fundamental semantic difficulties [30], and it is undesirable in applications where repeatability is important. 


\section{References}

1. Abadi, D.J., Ahmad, Y., Balazinska, M., Cetintemel, U., Cherniack, M., Hwang, J.H., Lindner, W., Maskey, A., Rasin, A., Ryvkina, E., Tatbul, N., Xing, Y., Zdonik, S.: The design of the Borealis stream processing engine. In: Proceedings of the 2nd Biennial Conference on Innovative Data Systems Research (CIDR '05). pp. 277-289 (2005), http://cidrdb.org/cidr2005/papers/P23.pdf

2. Abadi, D.J., Carney, D., Cetintemel, U., Cherniack, M., Convey, C., Lee, S., Stonebraker, M., Tatbul, N., Zdonik, S.: Aurora: A new model and architecture for data stream management. The VLDB Journal 12(2), 120-139 (2003). https://doi.org/10.1007/s00778-003-0095-z

3. Abbas, H., Alur, R., Mamouras, K., Mangharam, R., Rodionova, A.: Real-time decision policies with predictable performance. Proceedings of the IEEE, Special Issue on Design Automation for Cyber-Physical Systems 106(9), 1593-1615 (2018). https://doi.org/10.1109/JPROC.2018.2853608

4. Abbas, H., Rodionova, A., Mamouras, K., Bartocci, E., Smolka, S.A., Grosu, R.: Quantitative regular expressions for arrhythmia detection. IEEE/ACM Transactions on Computational Biology and Bioinformatics 16(5), 1586-1597 (2019). https://doi.org/10.1109/TCBB.2018.2885274

5. Affetti, L., Tommasini, R., Margara, A., Cugola, G., Della Valle, E.: Defining the execution semantics of stream processing engines. Journal of Big Data 4(1) (2017). https://doi.org/10.1186/s40537-017-0072-9

6. Akidau, T., Balikov, A., Bekiroğlu, K., Chernyak, S., Haberman, J., Lax, R., McVeety, S., Mills, D., Nordstrom, P., Whittle, S.: MillWheel: Fault-tolerant stream processing at Internet scale. Proceedings of the VLDB Endowment 6(11), 1033-1044 (2013). https://doi.org/10.14778/2536222.2536229

7. Akidau, T., Bradshaw, R., Chambers, C., Chernyak, S., Fernández-Moctezuma, R.J., Lax, R., McVeety, S., Mills, D., Perry, F., Schmidt, E., Whittle, S.: The dataflow model: A practical approach to balancing correctness, latency, and cost in massive-scale, unbounded, out-of-order data processing. Proceedings of the VLDB Endowment 8(12), 1792-1803 (2015). https://doi.org/10.14778/2824032.2824076

8. Alur, R., Cerný, P.: Streaming transducers for algorithmic verification of single-pass list-processing programs. In: Proceedings of the 38th Annual ACM SIGPLAN-SIGACT Symposium on Principles of Programming Languages. pp. 599-610. POPL '11, ACM, New York, NY, USA (2011). https://doi.org/10.1145/1926385.1926454

9. Alur, R., Fisman, D., Mamouras, K., Raghothaman, M., Stanford, C.: Streamable regular transductions. Theoretical Computer Science 807, 15-41 (2020). https://doi.org/10.1016/j.tcs.2019.11.018

10. Alur, R., Mamouras, K.: An introduction to the StreamQRE language. Dependable Software Systems Engineering 50, 1-24 (2017). https://doi.org/10.3233/9781-61499-810-5-1

11. Alur, R., Mamouras, K., Stanford, C.: Automata-based stream processing. In: Chatzigiannakis, I., Indyk, P., Kuhn, F., Muscholl, A. (eds.) Proceedings of the 44th International Colloquium on Automata, Languages, and Programming (ICALP '17). Leibniz International Proceedings in Informatics (LIPIcs), vol. 80, pp. 112:1-112:15. Schloss Dagstuhl-Leibniz-Zentrum fuer Informatik, Dagstuhl, Germany (2017). https://doi.org/10.4230/LIPIcs.ICALP.2017.112

12. Alur, R., Mamouras, K., Stanford, C.: Modular quantitative monitoring. Proceedings of the ACM on Programming Languages 3(POPL), 50:1-50:31 (2019). https://doi.org/10.1145/3290363 
13. Alur, R., Mamouras, K., Stanford, C., Tannen, V.: Interfaces for stream processing systems. In: Lohstroh, M., Derler, P., Sirjani, M. (eds.) Principles of Modeling: Essays Dedicated to Edward A. Lee on the Occasion of His 60th Birthday, Lecture Notes in Computer Science, vol. 10760, pp. 38-60. Springer, Cham (2018). https://doi.org/10.1007/978-3-319-95246-8_3

14. Alur, R., Mamouras, K., Ulus, D.: Derivatives of quantitative regular expressions. In: Aceto, L., Bacci, G., Bacci, G., Ingólfsdóttir, A., Legay, A., Mardare, R. (eds.) Models, Algorithms, Logics and Tools: Essays Dedicated to Kim Guldstrand Larsen on the Occasion of His 60th Birthday, Lecture Notes in Computer Science, vol. 10460, pp. 75-95. Springer, Cham (2017). https://doi.org/10.1007/978-3-31963121-9_4

15. Arasu, A., Babcock, B., Babu, S., Cieslewicz, J., Datar, M., Ito, K., Motwani, R., Srivastava, U., Widom, J.: STREAM: The Stanford data stream management system. Tech. Rep. 2004-20, Stanford InfoLab (2004), http://ilpubs.stanford.edu: $8090 / 641 /$

16. Arasu, A., Babu, S., Widom, J.: The CQL continuous query language: Semantic foundations and query execution. The VLDB Journal 15(2), 121-142 (2006). https://doi.org/10.1007/s00778-004-0147-z

17. Arasu, A., Widom, J.: A denotational semantics for continuous queries over streams and relations. SIGMOD Record 33(3), 6-11 (2004). https://doi.org/10.1145/1031570.1031572

18. Babcock, B., Babu, S., Datar, M., Motwani, R., Widom, J.: Models and issues in data stream systems. In: Proceedings of the Twenty-first ACM SIGMOD-SIGACT-SIGART Symposium on Principles of Database Systems. pp. 1-16. PODS '02, ACM, New York, NY, USA (2002). https://doi.org/10.1145/543613.543615

19. Bai, Y., Thakkar, H., Wang, H., Luo, C., Zaniolo, C.: A data stream language and system designed for power and extensibility. In: Proceedings of the 15th ACM International Conference on Information and Knowledge Management. pp. 337-346. CIKM '06, ACM, New York, NY, USA (2006). https://doi.org/10.1145/1183614.1183664

20. Benveniste, A., Caspi, P., Edwards, S.A., Halbwachs, N., Guernic, P.L., de Simone, R.: The synchronous languages 12 years later. Proceedings of the IEEE 91(1), 64-83 (2003). https://doi.org/10.1109/JPROC.2002.805826

21. Benveniste, A., Guernic, P.L., Jacquemot, C.: Synchronous programming with events and relations: The SIGNAL language and its semantics. Science of Computer Programming 16(2), 103-149 (1991). https://doi.org/10.1016/01676423(91)90001-E

22. Berry, G., Gonthier, G.: The Esterel synchronous programming language: Design, semantics, implementation. Science of Computer Programming 19(2), 87152 (1992). https://doi.org/10.1016/0167-6423(92)90005-V

23. Bertot, Y., Castéran, P.: Interactive Theorem Proving and Program Development. Springer (2013). https://doi.org/10.1007/978-3-662-07964-5

24. Bilsen, G., Engels, M., Lauwereins, R., Peperstraete, J.: Cyclo-static dataflow. IEEE Transactions on Signal Processing 44(2), 397-408 (1996). https://doi.org/10.1109/78.485935

25. Botan, I., Derakhshan, R., Dindar, N., Haas, L., Miller, R.J., Tatbul, N.: SECRET: A model for analysis of the execution semantics of stream processing systems. Proceedings of the VLDB Endowment 3(1-2), 232-243 (2010). https://doi.org/10.14778/1920841.1920874 
26. Bouillet, E., Kothari, R., Kumar, V., Mignet, L., Nathan, S., Ranganathan, A., Turaga, D.S., Udrea, O., Verscheure, O.: Processing 6 billion CDRs/day: From research to production (experience report). In: Proceedings of the 6th ACM International Conference on Distributed Event-Based Systems. pp. 264-267. DEBS '12, ACM, New York, NY, USA (2012). https://doi.org/10.1145/2335484.2335513

27. Bourke, T., Pouzet, M.: Zélus: A synchronous language with ODEs. In: Proceedings of the 16th International Conference on Hybrid Systems: Computation and Control. pp. 113-118. HSCC '13, ACM, New York, NY, USA (2013). https://doi.org/10.1145/2461328.2461348

28. Boussinot, F., de Simone, R.: The ESTEREL language. Proceedings of the IEEE 79(9), 1293-1304 (1991). https://doi.org/10.1109/5.97299

29. Brenna, L., Demers, A., Gehrke, J., Hong, M., Ossher, J., Panda, B., Riedewald, M., Thatte, M., White, W.: Cayuga: A high-performance event processing engine. In: Proceedings of the 2007 ACM SIGMOD International Conference on Management of Data. pp. 1100-1102. SIGMOD '07, ACM, New York, NY, USA (2007). https://doi.org/10.1145/1247480.1247620

30. Brock, J.D., Ackerman, W.B.: Scenarios: A model of non-determinate computation. In: Díaz, J., Ramos, I. (eds.) Proceedings of the International Colloquium on the Formalization of Programming Concepts (ICFPC '81). Lecture Notes in Computer Science, vol. 107, pp. 252-259. Springer, Berlin, Heidelberg (1981). https://doi.org/10.1007/3-540-10699-5_102

31. Caspi, P., Pilaud, D., Halbwachs, N., Plaice, J.A.: LUSTRE: A declarative language for real-time programming. In: Proceedings of the 14th ACM SIGACT-SIGPLAN Symposium on Principles of Programming Languages. pp. 178-188. POPL '87, ACM, New York, NY, USA (1987). https://doi.org/10.1145/41625.41641

32. Chandrasekaran, S., Cooper, O., Deshpande, A., Franklin, M.J., Hellerstein, J.M., Hong, W., Krishnamurthy, S., Madden, S., Raman, V., Reiss, F., Shah, M.: TelegraphCQ: Continuous dataflow processing for an uncertain world. In: Proceedings of the First Biennial Conference on Innovative Data Systems Research (CIDR '03) (2003), http://cidrdb.org/cidr2003/program/p24.pdf

33. Chen, C.M., Agrawal, H., Cochinwala, M., Rosenbluth, D.: Stream query processing for healthcare bio-sensor applications. In: Proceedings of the 20th International Conference on Data Engineering. pp. 791-794. ICDE '04, IEEE (2004). https://doi.org/10.1109/ICDE.2004.1320048

34. Cooper, G.H., Krishnamurthi, S.: Embedding dynamic dataflow in a call-by-value language. In: Sestoft, P. (ed.) Proceedings of the 15th European Symposium on Programming (ESOP '06). Lecture Notes in Computer Science, vol. 3924, pp. 294308. Springer, Berlin, Heidelberg (2006). https://doi.org/10.1007/11693024_20

35. Coquand, T., Huet, G.: The calculus of constructions. Information and Computation 76(2), 95-120 (1988). https://doi.org/10.1016/0890-5401(88)90005-3

36. Courtney, A.: Frappé: Functional reactive programming in Java. In: Ramakrishnan, I.V. (ed.) Proceedings of the 3rd International Symposium on Practical Aspects of Declarative Languages (PADL '01). Lecture Notes in Computer Science, vol. 1990, pp. 29-44. Springer, Berlin, Heidelberg (2001). https://doi.org/10.1007/3-540-45241-9_3

37. Cranor, C., Johnson, T., Spataschek, O., Shkapenyuk, V.: Gigascope: A stream database for network applications. In: Proceedings of the 2003 ACM SIGMOD International Conference on Management of Data. pp. 647-651. SIGMOD '03, ACM, New York, NY, USA (2003). https://doi.org/10.1145/872757.872838 
38. Czaplicki, E., Chong, S.: Asynchronous functional reactive programming for GUIs. In: Proceedings of the 34th ACM SIGPLAN Conference on Programming Language Design and Implementation. pp. 411-422. PLDI '13, ACM, New York, NY, USA (2013). https://doi.org/10.1145/2491956.2462161

39. D'Angelo, B., Sankaranarayanan, S., Sanchez, C., Robinson, W., Finkbeiner, B., Sipma, H.B., Mehrotra, S., Manna, Z.: LOLA: Runtime monitoring of synchronous systems. In: Proceedings of the 12th International Symposium on Temporal Representation and Reasoning (TIME '05). pp. 166-174. IEEE (2005). https://doi.org/10.1109/TIME.2005.26

40. Demers, A., Gehrke, J., Hong, M., Riedewald, M., White, W.: Towards expressive publish/subscribe systems. In: Ioannidis, Y., Scholl, M.H., Schmidt, J.W., Matthes, F., Hatzopoulos, M., Boehm, K., Kemper, A., Grust, T., Boehm, C. (eds.) Proceedings of the 10th International Conference on Extending Database Technology (EDBT '06). Lecture Notes in Computer Science, vol. 3896, pp. 627644. Springer, Berlin, Heidelberg (2006). https://doi.org/10.1007/11687238_38

41. Demers, A., Gehrke, J., Panda, B., Riedewald, M., Sharma, V., White, W.: Cayuga: A general purpose event monitoring system. In: Proceedings of the 3rd Biennial Conference on Innovative Data Systems Research (CIDR '07). pp. 412422 (2007), http://cidrdb.org/cidr2007/papers/cidr07p47.pdf

42. Dennis, J.B.: First version of a data flow procedure language. In: Robinet, B. (ed.) Programming Symposium. Lecture Notes in Computer Science, vol. 19, pp. 362-376. Springer, Berlin, Heidelberg (1974). https://doi.org/10.1007/3-54006859-7_145

43. Deshmukh, J.V., Donzé, A., Ghosh, S., Jin, X., Juniwal, G., Seshia, S.A.: Robust online monitoring of signal temporal logic. Formal Methods in System Design 51(1), 5-30 (2017). https://doi.org/10.1007/s10703-017-0286-7

44. Dindar, N., Tatbul, N., Miller, R.J., Haas, L.M., Botan, I.: Modeling the execution semantics of stream processing engines with SECRET. The VLDB Journal 22(4), 421-446 (2013). https://doi.org/10.1007/s00778-012-0297-3

45. Elgot, C.C., Mezei, J.E.: On relations defined by generalized finite automata. IBM Journal of Research and Development 9(1), 47-68 (1965). https://doi.org/10.1147/rd.91.0047

46. Elliott, C., Hudak, P.: Functional reactive animation. In: Proceedings of the Second ACM SIGPLAN International Conference on Functional Programming. pp. 263-273. ICFP '97, ACM, New York, NY, USA (1997). https://doi.org/10.1145/258948.258973

47. Elliott, C.M.: Push-pull functional reactive programming. In: Proceedings of the 2nd ACM SIGPLAN Symposium on Haskell. pp. 25-36. Haskell '09, ACM, New York, NY, USA (2009). https://doi.org/10.1145/1596638.1596643

48. Ginsburg, S., Rose, G.F.: A characterization of machine mappings. Canadian Journal of Mathematics 18, 381-388 (1966). https://doi.org/10.4153/CJM1966-040-3

49. Grathwohl, N.B.B., Kozen, D., Mamouras, K.: KAT + B! In: Proceedings of the Joint Meeting of the Twenty-Third EACSL Annual Conference on Computer Science Logic (CSL) and the Twenty-Ninth Annual ACM/IEEE Symposium on Logic in Computer Science (LICS). pp. 44:1-44:10. CSL-LICS '14, ACM, New York, NY, USA (2014). https://doi.org/10.1145/2603088.2603095

50. Gyllstrom, D., Wu, E., Chae, H.J., Diao, Y., Stahlberg, P., Anderson, G.: SASE: Complex event processing over streams. In: Proceedings of the 3rd Biennial Conference on Innovative Data Systems Research (CIDR '07). pp. 407-411 (2007), http://cidrdb.org/cidr2007/papers/cidr07p46.pdf 
51. Halbwachs, N., Caspi, P., Raymond, P., Pilaud, D.: The synchronous data flow programming language LUSTRE. Proceedings of the IEEE 79(9), 1305-1320 (1991). https://doi.org/10.1109/5.97300

52. Havelund, K., Roşu, G.: Efficient monitoring of safety properties. International Journal on Software Tools for Technology Transfer 6(2), 158-173 (2004). https://doi.org/10.1007/s10009-003-0117-6

53. Hirzel, M.: Partition and compose: Parallel complex event processing. In: Proceedings of the 6th ACM International Conference on Distributed EventBased Systems. pp. 191-200. DEBS '12, ACM, New York, NY, USA (2012). https://doi.org/10.1145/2335484.2335506

54. Hirzel, M., Soulé, R., Schneider, S., Gedik, B., Grimm, R.: A catalog of stream processing optimizations. ACM Computing Surveys (CSUR) 46(4), 46:1-46:34 (2014). https://doi.org/10.1145/2528412

55. Hudak, P., Courtney, A., Nilsson, H., Peterson, J.: Arrows, robots, and functional reactive programming. In: Jeuring, J., Jones, S.L.P. (eds.) Revised Lectures of the 4th International School on Advanced Functional Programming: AFP 2002, Oxford, UK, August 19-24, 2002., Lecture Notes in Computer Science, vol. 2638, pp. 159-187. Springer, Berlin, Heidelberg (2003). https://doi.org/10.1007/978-3540-44833-4_6

56. Hughes, J.: Generalising monads to arrows. Science of Computer Programming 37(1), 67-111 (2000). https://doi.org/10.1016/S0167-6423(99)00023-4

57. Jain, N., Mishra, S., Srinivasan, A., Gehrke, J., Widom, J., Balakrishnan, H., Çetintemel, U., Cherniack, M., Tibbetts, R., Zdonik, S.: Towards a streaming SQL standard. Proceedings of the VLDB Endowment 1(2), 1379-1390 (2008). https://doi.org/10.14778/1454159.1454179

58. Joyal, A., Street, R., Verity, D.: Traced monoidal categories. Mathematical Proceedings of the Cambridge Philosophical Society 119(3), 447--468 (1996). https://doi.org/10.1017/S0305004100074338

59. Kahn, G.: The semantics of a simple language for parallel programming. Information Processing 74, 471-475 (1974)

60. Kahn, G., MacQueen, D.B.: Coroutines and networks of parallel processes. Information Processing 77, 993-998 (1977)

61. Karp, R.M., Miller, R.E.: Properties of a model for parallel computations: Determinacy, termination, queueing. SIAM Journal on Applied Mathematics 14(6), 1390-1411 (1966). https://doi.org/10.1137/0114108

62. Kozen, D.: A completeness theorem for Kleene algebras and the algebra of regular events. Information and Computation 110(2), 366-390 (1994). https://doi.org/10.1006/inco.1994.1037

63. Kozen, D.: Kleene algebra with tests. ACM Transactions on Programming Languages and Systems (TOPLAS) 19(3), 427-443 (1997). https://doi.org/10.1145/256167.256195

64. Kozen, D., Mamouras, K.: Kleene algebra with equations. In: Esparza, J., Fraigniaud, P., Husfeldt, T., Koutsoupias, E. (eds.) Proceedings of the 41st International Colloquium on Automata, Languages and Programming (ICALP '14). Lecture Notes in Computer Science, vol. 8573, pp. 280-292. Springer, Berlin, Heidelberg (2014). https://doi.org/10.1007/978-3-662-43951-7_24

65. Kozen, D., Parikh, R.: An elementary proof of the completeness of PDL. Theoretical Computer Science 14(1), 113-118 (1981). https://doi.org/10.1016/03043975(81)90019-0 
66. Kozen, D., Tiuryn, J.: On the completeness of propositional Hoare logic. Information Sciences 139(3-4), 187-195 (2001). https://doi.org/10.1016/S00200255(01)00164-5

67. Krämer, J., Seeger, B.: Semantics and implementation of continuous sliding window queries over data streams. ACM Transactions on Database Systems (TODS) 34(1), 4:1-4:49 (2009). https://doi.org/10.1145/1508857.1508861

68. Krishnaswami, N.R.: Higher-order functional reactive programming without spacetime leaks. In: Proceedings of the 18th ACM SIGPLAN International Conference on Functional Programming. pp. 221-232. ICFP '13, ACM, New York, NY, USA (2013). https://doi.org/10.1145/2500365.2500588

69. Krishnaswami, N.R., Benton, N.: Ultrametric semantics of reactive programs. In: Proceedings of the 26th Annual IEEE Symposium on Logic in Computer Science (LICS '11). pp. 257-266. IEEE (2011). https://doi.org/10.1109/LICS.2011.38

70. Kulkarni, S., Bhagat, N., Fu, M., Kedigehalli, V., Kellogg, C., Mittal, S., Patel, J.M., Ramasamy, K., Taneja, S.: Twitter Heron: Stream processing at scale. In: Proceedings of the 2015 ACM SIGMOD International Conference on Management of Data. pp. 239-250. SIGMOD '15, ACM, New York, NY, USA (2015). https://doi.org/10.1145/2723372.2742788

71. Law, Y.N., Wang, H., Zaniolo, C.: Relational languages and data models for continuous queries on sequences and data streams. ACM Transactions on Database Systems (TODS) 36(2), 8:1-8:32 (2011). https://doi.org/10.1145/1966385.1966386

72. Le Guernic, P., Benveniste, A., Bournai, P., Gautier, T.: SIGNALa data flow-oriented language for signal processing. IEEE Transactions on Acoustics, Speech, and Signal Processing 34(2), 362-374 (1986). https://doi.org/10.1109/TASSP.1986.1164809

73. Lee, E.A., Messerschmitt, D.G.: Synchronous data flow. Proceedings of the IEEE 75(9), 1235-1245 (1987). https://doi.org/10.1109/PROC.1987.13876

74. Leucker, M., Schallhart, C.: A brief account of runtime verification. The Journal of Logic and Algebraic Programming 78(5), 293-303 (2009). https://doi.org/10.1016/j.jlap.2008.08.004

75. Li, J., Maier, D., Tufte, K., Papadimos, V., Tucker, P.A.: Semantics and evaluation techniques for window aggregates in data streams. In: Proceedings of the 2005 ACM SIGMOD International Conference on Management of Data. pp. 311-322. SIGMOD '05, ACM, New York, NY, USA (2005). https://doi.org/10.1145/1066157.1066193

76. Maier, D., Li, J., Tucker, P., Tufte, K., Papadimos, V.: Semantics of data streams and operators. In: Eiter, T., Libkin, L. (eds.) Proceedings of the 10th International Conference on Database Theory (ICDT '05). Lecture Notes in Computer Science, vol. 3363, pp. 37-52. Springer, Berlin, Heidelberg (2005). https://doi.org/10.1007/978-3-540-30570-5_3

77. Maier, I., Odersky, M.: Higher-order reactive programming with incremental lists. In: Castagna, G. (ed.) Proceedings of the 27th European Conference on Object-Oriented Programming (ECOOP '13). Lecture Notes in Computer Science, vol. 7920, pp. 707-731. Springer, Berlin, Heidelberg (2013). https://doi.org/10.1007/978-3-642-39038-8_29

78. Mamouras, K.: On the Hoare theory of monadic recursion schemes. In: Proceedings of the Joint Meeting of the 23rd EACSL Annual Conference on Computer Science Logic (CSL) and the 29th Annual ACM/IEEE Symposium on Logic in Computer Science (LICS). pp. 69:1-69:10. CSL-LICS '14, ACM, New York, NY, USA (2014). https://doi.org/10.1145/2603088.2603157 
79. Mamouras, K.: Extensions of Kleene Algebra for Program Verification. Ph.D. thesis, Cornell University, Ithaca, NY (August 2015), http://hdl.handle.net/1813/ 40960

80. Mamouras, K.: Synthesis of strategies and the Hoare logic of angelic nondeterminism. In: Pitts, A. (ed.) Proceedings of the 18th International Conference on Foundations of Software Science and Computation Structures (FoSSaCS '15). Lecture Notes in Computer Science, vol. 9034, pp. 25-40. Springer, Berlin, Heidelberg (2015). https://doi.org/10.1007/978-3-662-46678-0_2

81. Mamouras, K.: The Hoare logic of deterministic and nondeterministic monadic recursion schemes. ACM Transactions on Computational Logic (TOCL) 17(2), 13:1-13:30 (2016). https://doi.org/10.1145/2835491

82. Mamouras, K.: Synthesis of strategies using the Hoare logic of angelic and demonic nondeterminism. Logical Methods in Computer Science 12(3) (2016). https://doi.org/10.2168/LMCS-12(3:6)2016

83. Mamouras, K.: Equational theories of abnormal termination based on Kleene algebra. In: Esparza, J., Murawski, A.S. (eds.) Proceedings of the 20th International Conference on Foundations of Software Science and Computation Structures (FoSSaCS '17). Lecture Notes in Computer Science, vol. 10203, pp. 88-105. Springer, Berlin, Heidelberg (2017). https://doi.org/10.1007/978-3-662-54458-7_6

84. Mamouras, K., Raghothaman, M., Alur, R., Ives, Z.G., Khanna, S.: StreamQRE: Modular specification and efficient evaluation of quantitative queries over streaming data. In: Proceedings of the 38th ACM SIGPLAN Conference on Programming Language Design and Implementation. pp. 693-708. PLDI '17, ACM, New York, NY, USA (2017). https://doi.org/10.1145/3062341.3062369

85. Mamouras, K., Stanford, C., Alur, R., Ives, Z.G., Tannen, V.: Data-trace types for distributed stream processing systems. In: Proceedings of the 40th ACM SIGPLAN Conference on Programming Language Design and Implementation. pp. 670-685. PLDI '19, ACM, New York, NY, USA (2019). https://doi.org/10.1145/3314221.3314580

86. McSherry, F., Murray, D.G., Isaacs, R., Isard, M.: Differential dataflow. In: Proceedings of the 6th Biennial Conference on Innovative Data Systems Research (CIDR '13) (2013), http://cidrdb.org/cidr2013/Papers/CIDR13_Paper111.pdf

87. Mealy, G.H.: A method for synthesizing sequential circuits. The Bell System Technical Journal 34(5), 1045-1079 (1955). https://doi.org/10.1002/j.15387305.1955.tb03788.x

88. Mei, Y., Madden, S.: ZStream: A cost-based query processor for adaptively detecting composite events. In: Proceedings of the 2009 ACM SIGMOD International Conference on Management of Data. pp. 193-206. SIGMOD '09, ACM, New York, NY, USA (2009). https://doi.org/10.1145/1559845.1559867

89. Meyerovich, L.A., Guha, A., Baskin, J., Cooper, G.H., Greenberg, M., Bromfield, A., Krishnamurthi, S.: Flapjax: A programming language for Ajax applications. In: Proceedings of the 24th ACM SIGPLAN Conference on Object Oriented Programming Systems Languages and Applications. pp. 1-20. OOPSLA '09, ACM, New York, NY, USA (2009). https://doi.org/10.1145/1640089.1640091

90. Moore, E.F.: Gedanken-Experiments on Sequential Machines, Annals of Mathematics Studies, vol. 34, pp. 129-153. Princeton University Press (1956)

91. Motwani, R., Widom, J., Arasu, A., Babcock, B., Babu, S., Datar, M., Manku, G.S., Olston, C., Rosenstein, J., Varma, R.: Query processing, approximation, and resource management in a data stream management system. In: Proceedings of the First Biennial Conference on Innovative Data Systems Research (CIDR '03) (2003), http://cidrdb.org/cidr2003/program/p22.pdf 
92. Murray, D.G., McSherry, F., Isaacs, R., Isard, M., Barham, P., Abadi, M.: Naiad: A timely dataflow system. In: Proceedings of the Twenty-Fourth ACM Symposium on Operating Systems Principles. pp. 439-455. SOSP '13, ACM, New York, NY, USA (2013). https://doi.org/10.1145/2517349.2522738

93. Nilsson, H., Courtney, A., Peterson, J.: Functional reactive programming, continued. In: Proceedings of the 2002 ACM SIGPLAN Workshop on Haskell. pp. 51-64. Haskell '02, ACM, New York, NY, USA (2002). https://doi.org/10.1145/581690.581695

94. Noghabi, S.A., Paramasivam, K., Pan, Y., Ramesh, N., Bringhurst, J., Gupta, I., Campbell, R.H.: Samza: Stateful scalable stream processing at LinkedIn. Proceedings of the VLDB Endowment 10(12), 1634-1645 (2017). https://doi.org/10.14778/3137765.3137770

95. Raney, G.N.: Sequential functions. Journal of the ACM 5(2), 177-180 (1958). https://doi.org/10.1145/320924.320930

96. Rutten, J.J.M.M.: Automata and coinduction (an exercise in coalgebra). In: Sangiorgi, D., de Simone, R. (eds.) Proceedings of the 9th International Conference on Concurrency Theory (CONCUR '98). Lecture Notes in Computer Science, vol. 1466, pp. 194-218. Springer, Berlin, Heidelberg (1998). https://doi.org/10.1007/BFb0055624

97. Rutten, J.J.M.M.: Universal coalgebra: A theory of systems. Theoretical Computer Science 249(1), 3-80 (2000). https://doi.org/10.1016/S03043975(00)00056-6

98. Rutten, J.J.M.M.: A coinductive calculus of streams. Mathematical Structures in Computer Science 15(1), 93-147 (2005). https://doi.org/10.1017/S0960129504004517

99. Sadri, R., Zaniolo, C., Zarkesh, A., Adibi, J.: Expressing and optimizing sequence queries in database systems. ACM Transactions on Database Systems 29(2), 282318 (2004). https://doi.org/10.1145/1005566.1005568

100. Sakarovitch, J.: Elements of Automata Theory. Cambridge University Press (2009)

101. Schneider, S., Hirzel, M., Gedik, B., Wu, K.L.: Safe data parallelism for general streaming. IEEE Transactions on Computers 64(2), 504-517 (2015). https://doi.org/10.1109/TC.2013.221

102. Schützenberger, M.P.: Sur une variante des fonctions séquentielles. Theoretical Computer Science 4(1), 47-57 (1977). https://doi.org/10.1016/03043975(77)90055-X

103. Sculthorpe, N., Nilsson, H.: Safe functional reactive programming through dependent types. In: Proceedings of the 14th ACM SIGPLAN International Conference on Functional Programming. pp. 23-34. ICFP '09, ACM, New York, NY, USA (2009). https://doi.org/10.1145/1596550.1596558

104. Shivers, O., Might, M.: Continuations and transducer composition. In: Proceedings of the 27th ACM SIGPLAN Conference on Programming Language Design and Implementation. pp. 295-307. PLDI '06, ACM, New York, NY, USA (2006). https://doi.org/10.1145/1133981.1134016

105. Thati, P., Roşu, G.: Monitoring algorithms for metric temporal logic specifications. Electronic Notes in Theoretical Computer Science 113, 145-162 (2005). https://doi.org/10.1016/j.entcs.2004.01.029

106. The Coq development team: The Coq proof assistant. https://coq.inria.fr (2020), [Online; accessed February 22, 2020] 
107. Thies, W., Karczmarek, M., Amarasinghe, S.: StreamIt: A language for streaming applications. In: Horspool, R.N. (ed.) Proceedings of the 11th International Conference on Compiler Construction (CC '02). Lecture Notes in Computer Science, vol. 2304, pp. 179-196. Springer, Berlin, Heidelberg (2002). https://doi.org/10.1007/3-540-45937-5_14

108. Toshniwal, A., Taneja, S., Shukla, A., Ramasamy, K., Patel, J.M., Kulkarni, S., Jackson, J., Gade, K., Fu, M., Donham, J., Bhagat, N., Mittal, S., Ryaboy, D.: Storm @ Twitter. In: Proceedings of the 2014 ACM SIGMOD International Conference on Management of Data. pp. 147-156. SIGMOD '14, ACM, New York, NY, USA (2014). https://doi.org/10.1145/2588555.2595641

109. Tucker, P.A., Maier, D., Sheard, T., Fegaras, L.: Exploiting punctuation semantics in continuous data streams. IEEE Transactions on Knowledge and Data Engineering 15(3), 555-568 (2003). https://doi.org/10.1109/TKDE.2003.1198390

110. Veanes, M., Hooimeijer, P., Livshits, B., Molnar, D., Bjorner, N.: Symbolic finite state transducers: Algorithms and applications. In: Proceedings of the 39th Annual ACM SIGPLAN-SIGACT Symposium on Principles of Programming Languages. pp. 137-150. POPL '12, ACM, New York, NY, USA (2012). https://doi.org/10.1145/2103656.2103674

111. Wu, E., Diao, Y., Rizvi, S.: High-performance complex event processing over streams. In: Proceedings of the 2006 ACM SIGMOD International Conference on Management of Data. pp. 407-418. SIGMOD '06, ACM, New York, NY, USA (2006). https://doi.org/10.1145/1142473.1142520

112. Zaharia, M., Das, T., Li, H., Hunter, T., Shenker, S., Stoica, I.: Discretized streams: Fault-tolerant streaming computation at scale. In: Proceedings of the Twenty-Fourth ACM Symposium on Operating Systems Principles. pp. 423-438. SOSP '13, ACM, New York, NY, USA (2013). https://doi.org/10.1145/2517349.2522737

113. Zaharia, M., Xin, R.S., Wendell, P., Das, T., Armbrust, M., Dave, A., Meng, X., Rosen, J., Venkataraman, S., Franklin, M.J., Ghodsi, A., Gonzalez, J., Shenker, S., Stoica, I.: Apache Spark: A unified engine for big data processing. Communications of the ACM 59(11), 56-65 (2016). https://doi.org/10.1145/2934664

Open Access This chapter is licensed under the terms of the Creative Commons Attribution 4.0 International License (http://creativecommons.org/licenses/by/4.0/), which permits use, sharing, adaptation, distribution and reproduction in any medium or format, as long as you give appropriate credit to the original author(s) and the source, provide a link to the Creative Commons license and indicate if changes were made.

The images or other third party material in this chapter are included in the chapter's Creative Commons license, unless indicated otherwise in a credit line to the material. If material is not included in the chapter's Creative Commons license and your intended use is not permitted by statutory regulation or exceeds the permitted use, you will need to obtain permission directly from the copyright holder. 